\title{
Polarization Calibration of the Helioseismic and Magnetic Imager (HMI) onboard the Solar Dynamics Observatory (SDO)
}

\author{
J. Schou • J.M. Borrero • A.A. Norton • S. Tomczyk • \\ D. Elmore · G.L. Card
}

Received: 29 May 2010 / Accepted: 8 September 2010 / Published online: 30 October 2010

(C) The Author(s) 2010. This article is published with open access at Springerlink.com

\begin{abstract}
As part of the overall ground-based calibration of the Helioseismic and Magnetic Imager (HMI) instrument an extensive set of polarimetric calibrations were performed. This paper describes the polarimetric design of the instrument, the test setup, the polarimetric model, the tests performed, and some results. It is demonstrated that HMI achieves an accuracy of $1 \%$ or better on the crosstalks between $Q, U$, and $V$ and that our model can reproduce the intensities in our calibration sequences to about $0.4 \%$. The amount of depolarization is negligible when the instrument is operated as intended which, combined with the flexibility of the polarimeter design, means that the polarimetric efficiency is excellent.
\end{abstract}

The Solar Dynamics Observatory

Guest Editors: W. Dean Pesnell, Phillip C. Chamberlin, and Barbara J. Thompson.

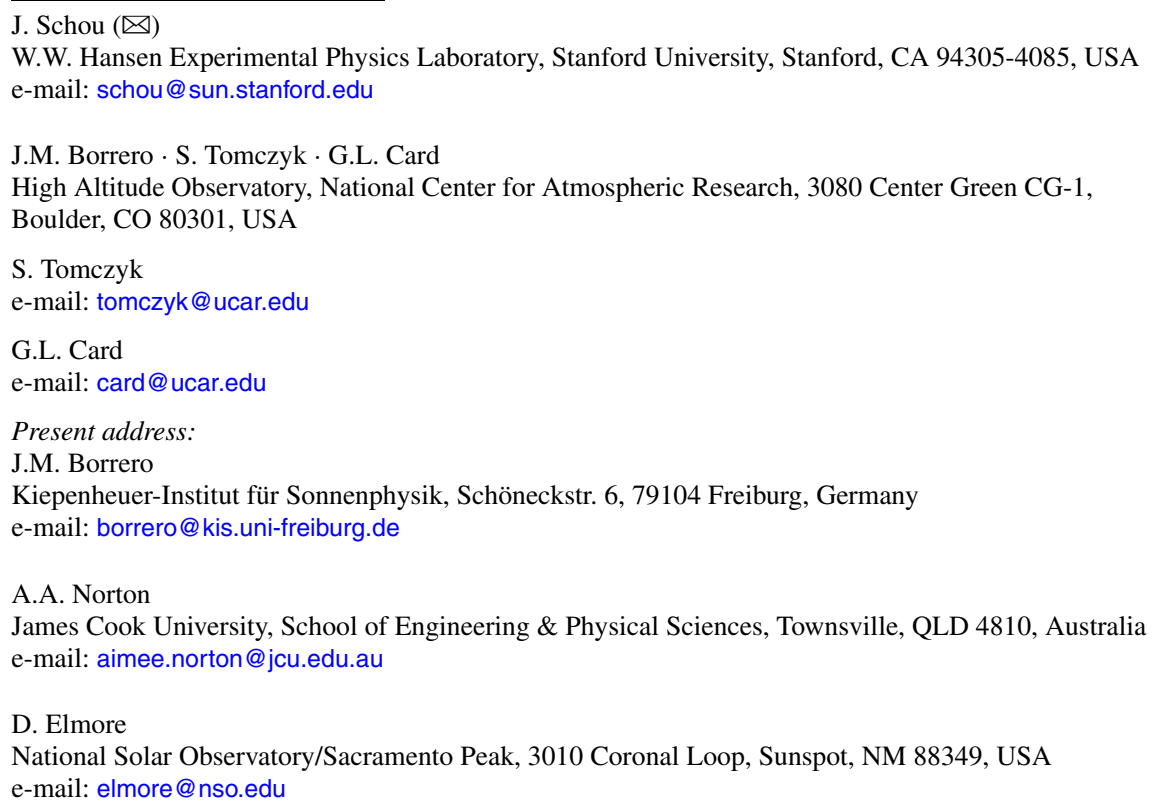


Keywords Instrumentation and data management $\cdot$ Magnetic fields, photosphere $\cdot$ Solar Dynamics Observatory

\section{Introduction}

Precise polarimetry requires the calibration of the polarimeter and associated telescope. Polarimeter calibration is typically performed by measuring the response of the polarimeter to known polarization states created by calibration optics placed at the output of the telescope (Skumanich et al., 1997; Beck et al., 2005). Telescope polarization is measured by placing polarizers over the entrance aperture of the telescope (Beck et al., 2005; Selbing, 2005) or by using the symmetry and anti-symmetry expected, or more precisely, not expected, in spectral Stokes profiles in Zeeman-sensitive lines (Collados, 2003; Kuhn et al., 1994).

In-flight calibration optics were avoided on the Helioseismic and Magnetic Imager (HMI) instrument, as they were on Hinode, due to the risk of a mechanical failure leaving a calibration optic in the beam and also for complexity, mass, and power considerations. Absence of calibration optics limits the types of calibrations that can be performed after the instrument has been placed into operation and places particular importance on ensuring that groundbased calibrations are comprehensive and consider the range of possible environmental and instrumental drifts.

As with the Hinode ground-based calibration (Ichimoto et al., 2008), the HMI polarimeter and telescope are calibrated together by placing calibration optics in front of the telescope and treating the entire optical path as the polarimeter.

The HMI instrument differs from most other instruments by having redundant polarization selectors (see Section 2.3). This was done to improve the reliability but has the consequence that it is necessary to derive a calibration valid for all settings of the polarization selectors rather than only for the expected combinations.

The design of observing sequences is discussed by Borrero et al. (2007).

As a matter of convention, the angles in the system are measured counterclockwise from horizontal as viewed from behind the instrument toward the Sun, with horizontal being the plane in which the light travels from the window through the filter system, i.e. the plane at which the cut in Figure 1 is made (see Sections 2 and 5.1.1). Horizontal polarization is $I+Q$, while $I+U$ corresponds to polarization at a 45 degree counterclockwise angle from horizontal, again as seen from the instrument.

The remainder of this section contains a brief description of the requirements of the instrument. Sections 2 and 3 contain descriptions of the instrument with an emphasis on the polarimetric aspects and the test setup, respectively. Section 4 contains a description of the polarimetric model used to describe the instrument and test equipment. Section 5 contains descriptions of the various tests and the analysis, while Section 6 describes the main results obtained. Section 7 describes how the results obtained will be used to calibrate the instrument, the accuracy achieved, and the plans for on-orbit calibrations. Section 8 presents the conclusion. In the appendices, details of the polarimetric models of different types of optical elements are given and the sequences run are described.

\subsection{Requirements}

HMI measures a linear combination $L_{\mathrm{HMI}}$ of the solar Stokes vector:

$$
L_{\mathrm{HMI}}=\mathcal{O} I_{\mathrm{Sun}},
$$

where $\mathcal{O}$ is the modulation matrix. In order to demodulate and obtain the Stokes vector, the demodulation matrix $[\mathcal{D}]$ must be known and applied: 


$$
I_{\mathrm{HMI}}=\mathcal{D} L_{\mathrm{HMI}}=\mathcal{D O} I_{\text {Sun }} .
$$

In reality there are systematic errors and the error matrix $\mathcal{E}=\mathcal{D} \mathcal{O}_{\text {true }}-\mathcal{I}$, where $\mathcal{O}_{\text {true }}$ is the actual modulation matrix and $\mathcal{I}$ the identity matrix, will not be identically zero.

To derive a requirement for maximum allowable elements in $\mathcal{E}$, it may be noted that the requirement on the noise in $Q, U$, and $V$ is $0.3 \%$ of $I$ in ten minutes and that systematic errors of less than $0.1 \%$ of $I$ on $Q, U$, and $V$ will thus only result in a marginal increase in the overall error. With typical values of $Q / I, U / I$, and $V / I$ of around 0.1 , it thus follows that the cross terms between $Q, U$, and $V$ of less than 0.01 are desirable. From $I$ to $Q, U$, and $V$ values less than 0.001 are needed. The terms into $I$ are less critical as they more or less correspond to a flat-field error and a total contribution of 0.01 should be adequate. This results in a requirement that the absolute values of $\mathcal{E}$ should be less than

$$
\mathcal{E}_{\max }=\left(\begin{array}{cccc}
0.01 & 0.1 & 0.1 & 0.1 \\
0.001 & 0.01 & 0.01 & 0.01 \\
0.001 & 0.01 & 0.01 & 0.01 \\
0.001 & 0.01 & 0.01 & 0.01
\end{array}\right)
$$

To be able to construct left- and right-circular polarization [LCP and RCP] from a single camera and to keep a 50-second or better overall cadence for the Doppler and Line-of-Sight (LOS) field, a requirement was imposed to be able to derive $V$ with less than $5 \%$ leakage from each of $Q$ and $U$, across the field of view using only the motor positions available (see Sections 2.3 and 7.2.4).

Some of the measurements required to finally verify some of these requirements are deferred to on-orbit operations. This is discussed in Section 7.3.1.

How errors relate to magnetic-field properties is discussed by Norton et al. (2006).

\section{Instrument Description}

The overall layout of the instrument is described by Schou et al. (2010). The parts of the instrument relevant to the polarimetry are shown in Figure 1. In order, the light passes through a front window, the primary lens, the secondary lens, a focus block (or calibration lens), three polarization-selector waveplates (PS1, PS2, and PS3), an Image Stabilization System [ISS] fold mirror, another focus block (or calibration lens), and a polarizing beamsplitter, all of which are described below. Following these elements are a Lyot filter, two Michelson interferometers, and a beamsplitter dividing the light to a pair of shutters and cameras (front and side) for higher cadence and redundancy. It may be noted that since the light is essentially monochromatic, the instrument is not required to be achromatic.

\subsection{Telescope}

The telescope consists of the front window, primary, and secondary lenses. The front window and primary are very close to a pupil and are thus effectively indistinguishable. The secondary lens is not at the same equivalent position, but we have nonetheless chosen to treat the telescope as a unit.

The front window is a sandwich consisting of $6 \mathrm{~mm}$ of BK7G18 radiation-hard glass, $3 \mathrm{~mm}$ of GG495 green glass, and $6 \mathrm{~mm}$ of BK7G18 glass. There are two heaters on the mounting ring, allowing the temperature to be controlled. Given the poor thermal conductivity of glass this, of course, does not ensure that the rest of the window is at any particular temperature, although it will eventually reach a nearly steady state with a slowly varying radial temperature gradient. 


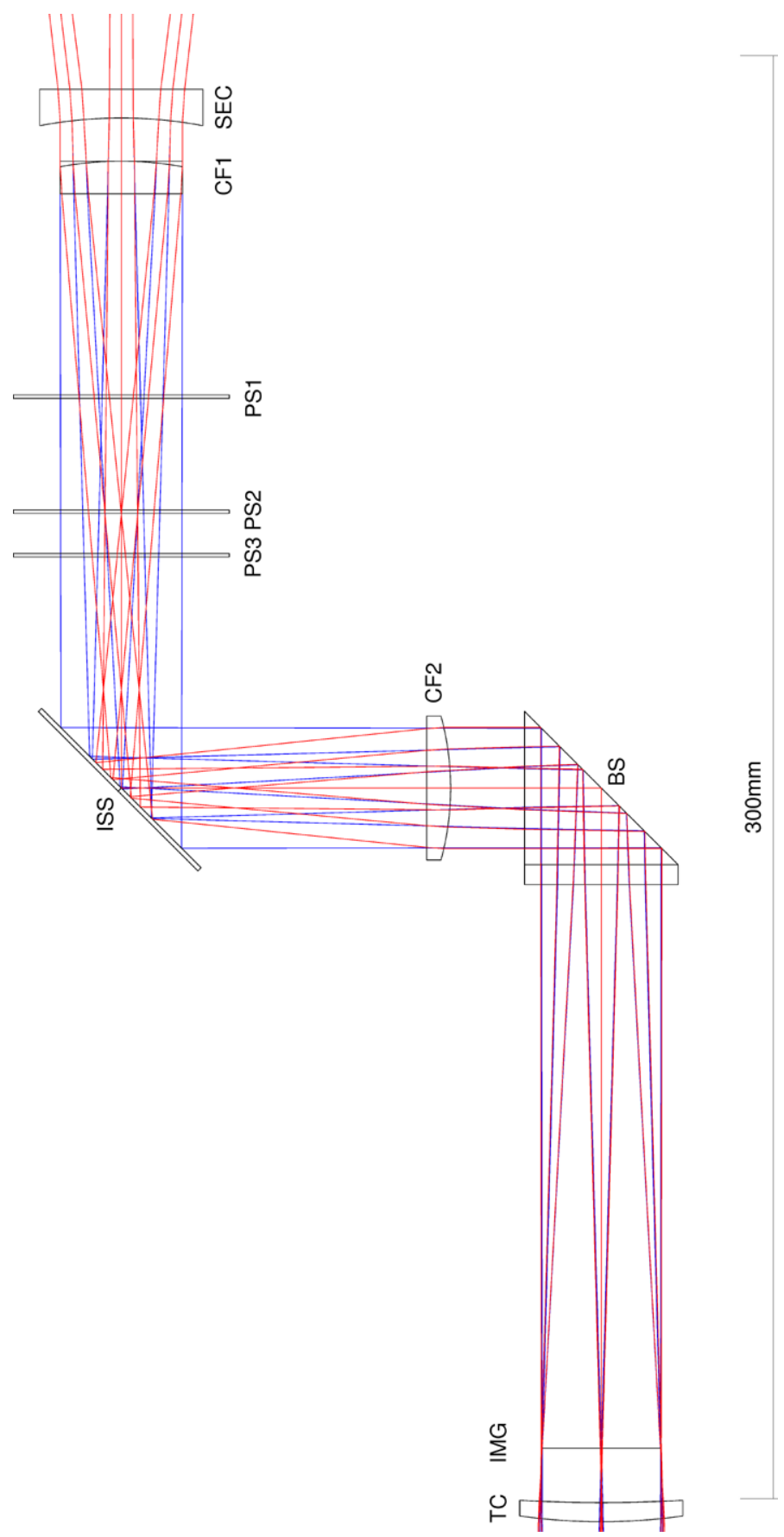

Figure 1 Raytrace with observing mode (obsmode) rays shown in blue and calibration mode (calmode) (see Section 2.2) rays shown in red. In the order that the light travels, the elements shown are: Secondary lens (SEC), first calibration lens (CF1), three polarization selectors (PS1, PS2, and PS3), ISS fold mirror (ISS), second calibration lens (CF2), polarizing beamsplitter (BS), a line showing the primary focus (IMG), and telecentric lens (TC). 
The primary lens is coupled both conductively and radiatively to the front window and to the separately temperature-controlled telescope tube and is thus fairly well controlled, but will see some temperature gradients.

Some effects of the temperature gradients are discussed in Section 6.2.

\subsection{Focus Blocks}

The focus blocks are mounted on two filter wheels and different thicknesses of glass (including zero) can be inserted to adjust the overall focus. It is also possible to replace each focus block with a lens causing the entrance pupil to be imaged onto the detector. This mode is known as calibration mode (calmode), while the regular mode is referred to as observation mode (obsmode).

As shown by the ray trace in Figure 1, the introduction of the calibration lenses dramatically changes the angles of incidence through the polarization selectors, a subject that will be discussed in more detail in Section 4.4.

\subsection{Polarization Selectors}

The polarization selectors are high-order-crystal quartz waveplates mounted in hollow core motors (HCMs) capable of rotating each waveplate to 240 equally spaced positions. The choice of high-order waveplates was made to avoid mechanical problems associated with low-order waveplates, but it does have some implications, as discussed in Section 4.4.

The three waveplates have nominal retardances of 10.50, 10.25, and 10.50 waves, in the order encountered. Using three waveplates allows for some amount of redundancy. A study of possible combinations of waveplates revealed that several combinations achieve full three for two redundancy, meaning that any one of the three waveplates can be stuck at any position while still allowing for the measurement of an arbitrary polarization state (limited, of course, by the coarseness of the angular settings and any inaccuracies in the retardances). Of these combinations, two involve only simple retardances: $(1 / 2,1 / 4,1 / 2)$ and $(1 / 2,1 / 4,1 / 4)$. These result in equal motion of the HCMs (and thus presumably wear) through a standard observing sequence with the optimal choice of which waveplate (the middle) to keep fixed. The former causes slightly less wear in case one of the other waveplates cannot be rotated, so it was chosen.

For waveplates 1 and 2, increasing motor steps correspond to clockwise (dir $=-1$ ) rotation of the waveplates as seen from the back of the instrument toward the Sun. For waveplate 3 the motion is counterclockwise $(\mathrm{dir}=+1)$. In all cases one motor step is $1.5^{\circ}$. It thus follows that the angle of the fast axis of the waveplate (relative to horizontal) is given by $C_{i}-\theta_{i}$, where $C_{i}=1.5^{\circ} \times \operatorname{dir}_{i} \times \mathrm{N}_{i}, \mathrm{~N}_{i}$ is the commanded motor position, and $\theta_{i}$ is the angle at which the fast axis is horizontal. The algorithm by which the motors are commanded is given in Schou et al. (2010).

During the assembly of the instrument, the motor positions at which the fast axis of each waveplate is horizontal were measured to be roughly 62,89 , and 9 . It thus follows that roughly $\theta_{1}=-93^{\circ}, \theta_{2}=-133.5^{\circ}$, and $\theta_{3}=13.5^{\circ}$. These values are unlikely to be accurate to better than one or two steps.

\subsection{Polarizing Beamsplitter and ISS Mirror}

The ISS mirror is used to stabilize the images and has essentially no effect from a polarimetric point of view (see Sections 4.5 and 7.1). 
The polarizing beamsplitter selects a linear polarization state (roughly vertical) to measure from the light entering it and directs it to the Lyot filter. The orthogonally polarized light goes to the limb sensor (not shown in Figure 1). The choice of a polarizing beamsplitter (as opposed to a polarizer) causes a field dependence of the direction of this linear state, as discussed in Section 4.5. To avoid effects on the filter profiles of this and of any Lyot filter to beamsplitter rotational misalignment, a cleanup polarizer after the telecentric lens ensures that the direction of polarization delivered to the Lyot filter is unchanging and uniform.

\section{Test Setup}

The test setup consists of a stimulus telescope followed by a polarization calibration unit (PCU) described below, which allows for the generation of various states of polarized light.

The stimulus telescope, which is described in more detail by Schou et al. (2010) and Wachter et al. (2010), consists of a reversed HMI telescope (secondary plus primary lens), an optional target at the prime focus, and a light source. The light source consists of a fiber bundle, one end of which is fed by a stabilized lamp and the other end of which is imaged through the focus of the stimulus telescope onto the pupil. This setup results in a uniform illumination of both the pupil and the image with very little polarization (see Section 6.1.1).

Sunlight was also used, but the results are not discussed here as the weak but rotating polarization caused by the heliostat mirrors causes artifacts. The vacuum tests performed are not discussed due to artifacts introduced by the vacuum-chamber window.

\subsection{Polarization Calibration Unit}

The PCU, designed by the High Altitude Observatory, consists of a dichroic polymer linear polarizer followed by a roughly quarter-waveplate zero-order plastic retarder, both of which are mounted on fused-silica substrates. Each element can be inserted (or not) into the light beam and rotated (see Figure 2) to a commanded angle.

The commanded rotation angle at which the polarization passed by the polarizer is horizontal was precisely determined by an independent measurement to be $-5.8^{\circ}$ with an uncertainty of about $0.2^{\circ}$. The polarizer rotates clockwise (negative in angle) when commanded to rotate in the positive direction. The PCU-to-instrument alignment is discussed in Section 5.1.1

The retarder was determined to have the fast axis horizontal at a commanded angle of $\theta_{\text {ret }}=90.6^{\circ}$ (also with an uncertainty of about $0.2^{\circ}$ ) and rotates counterclockwise (positive in angle) when commanded to rotate in the positive direction. However, a correction to this angle is determined as part of the calibration, and so the accuracy of this value is of little consequence, except as a consistency check.

\section{Polarimetric Model}

The light emitted by the stimulus telescope $\left[I_{\text {stim }}=(I, Q, U, V)\right]$ goes through the PCU and HMI, being finally detected at the CCD as $L_{\text {cal }}$ :

$$
L_{\text {cal }}=\mathcal{O} \times \mathcal{M}_{\text {pcu }} \times I_{\text {stim }}=\left(P_{\text {det }}^{T} \times \mathcal{M}_{\text {sel }} \times \mathcal{M}_{\text {tel }}\right) \times \mathcal{M}_{\text {pcu }} \times I_{\text {stim }}
$$

where $\mathcal{O}$ is the modulation matrix given by Equation (1) and the matrices $\mathcal{M}_{\mathrm{pcu}}, \mathcal{M}_{\text {sel }}$, and $\mathcal{M}_{\text {tel }}$ are the Mueller matrices for the PCU, polarization selectors, and telescope, respectively. $P_{\text {det }}$ gives the Stokes vector passed by the polarizing beamsplitter, i.e. the first row of 


\section{HMI PQLARIZATION CALIBRATION UNIT}

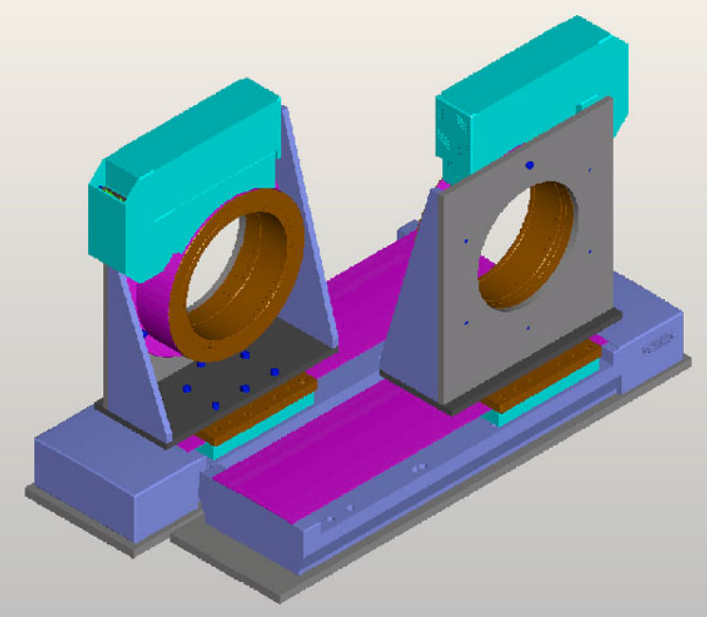

Figure 2 Schematic of the PCU. Each unit consists of a linear stage and a rotary stage with a piece of optics. The stage closest to the stimulus telescope contains a polarizer while the stage closest to the instrument contains a retarder. Otherwise, the two units are identical. The optics are removable and the polarizer unit can be fitted with black plates with aperture stops, as discussed in Section 5.1.1. In normal operation, the stages are enclosed in boxes for protection and to provide baffling of stray light.

the Mueller matrix of a polarizer. The dependency of the various components on the field position, instrument, and PCU settings has been suppressed for simplicity. Unless noted, the center ray hitting a given pixel is used and no integration over incidence angle is performed.

We present below details of the modeling of the components. In some cases, different models taking into account various physical effects and imperfections have been used. Further details of the models of various types of optical elements are given in Appendix A.

\subsection{Stimulus Telescope}

The stimulus-telescope polarization vector does not need any special modeling and simply has four components of a vector $(I, Q, U, V)$.

\section{2. $\mathrm{PCU}$}

The PCU is modeled as a polarizer followed by a retarder, but several effects need to be taken into account. First of all both elements have a transmission less than $100 \%$.

$$
\mathcal{M}_{\mathrm{pcu}}=t_{\mathrm{ret}} W\left(\mathrm{C}_{\mathrm{ret}}-\theta_{\mathrm{ret}}, r_{\mathrm{ret}}\right) \times P\left(C_{\mathrm{pol}}, t_{\mathrm{pol}}\right)
$$

where $W$ and $P$ are given in Appendices A.3 and A.1, respectively, $t_{\text {ret }}$ is the retarder transmission, $\mathrm{C}_{\text {ret }}$ the commanded retarder rotation angle (corrected for the known offset discussed in Section 3.1), $\theta_{\text {ret }}$ is the retarder angle at which the fast axis is horizontal, $r_{\text {ret }}$ the retardance, and $C_{\mathrm{pol}}$ is the polarizer rotation angle (again corrected for the known offset). 
The two rotation angles $\left[\mathrm{C}_{\text {ret }}\right.$ and $\left.C_{\mathrm{pol}}\right]$ are known from the PCU commanding (and telemetry) and thus vary from position to position in the test sequences. The rest of the parameters $\left[t_{\text {ret }}, \theta_{\text {ret }}, r_{\text {ret }}\right.$, and $\left.t_{\text {pol }}\right]$ are fitted. There is no fitted offset for the polarizer as it is used as the absolute coordinate reference. When a component is out of the beam, the corresponding Mueller matrix is replaced by the identity matrix.

Both PCU elements have significant wavefront errors and thus introduce distortions of the stimulus-telescope image which, due to the non-uniform illumination, cause intensity changes unrelated to the polarization. We have found that the main errors are those caused by the image shifts. To model those we multiply the overall intensity by a factor

$$
I_{\mathrm{PCU}}=\left(1+\Delta x I_{x}\right) \times\left(1+\Delta y I_{y}\right),
$$

where $\Delta x$ and $\Delta y$ are the image offsets and $I_{x}$ and $I_{y}$ the (fitted) intensity gradients. The image offsets are separately determined by installing a field stop, tracking the edge, and fitting the results to a simple sinusoidal variation with retarder and polarizer rotation angle. Since the motions are repeatable, this is not done for every sequence run.

This effect is not included in calmode since the PCU elements are near a pupil.

Changes in the overall image size caused by wavefront errors (and the resulting change in intensity) are captured in the element transmissions.

\subsection{Front Window and Telescope}

Two basic models have been used for the front window. In the first the window is modeled as a general six-term Mueller matrix $\left(\mathcal{M}_{\text {tel }}=M_{6}\right.$ in Appendix A.3), allowing for both retardance and depolarization. The second uses a combination of a perfect retarder and a partial depolarizer: $\mathcal{M}_{\text {tel }}=D_{3} \times W_{M}$, where $D_{3}$ is the depolarizer described in Appendix A.2 and $W_{M}$ is described by specifying $M_{Q V}$ and $M_{U V}$ (see Appendix A.4).

\subsection{Polarization Selectors}

The polarization selectors would ideally be represented by $W(\theta, r)$ (see Equation (11) in Appendix A.3). However, they are high order and the incidence angles are significant, especially in calmode (see Figure 1), and thus a model with incidence angle $W_{I}(\theta, r, \alpha, \beta)$ (see Equation (12) in Appendix A.3) is used.

$$
\mathcal{M}_{\text {sel }}=W_{I}\left(C_{3}-\theta_{3}, r_{3}, \alpha, \beta\right) \times W_{I}\left(C_{2}-\theta_{2}, r_{2}, \alpha, \beta\right) \times W_{I}\left(C_{1}-\theta_{1}, r_{1}, \alpha, \beta\right),
$$

where the suffix is the selector number in the order encountered, $C_{i}$ is the commanded motor position, $\theta_{i}$ is the position at which the fast axis is horizontal, $r_{i}$ is the retardance, $\alpha$ is the incidence angle, and $\beta$ is the angle from horizontal between the center of the field of view and the point of interest. Of these $C_{i}, \alpha$, and $\beta$ are known from commanding or optical ray traces, while $\theta_{i}$ and $r_{i}$ are fitted for. Note that the $r_{i}$ have to be the true retardances (e.g. 10.50 , not 0.50 ) and that $\alpha$ is different in obsmode and calmode (see red and blue rays in Figure 1). Transmissions are assumed to be 1.0 since the waveplates are always in the beam.

As discussed in Section 5.1 the $\theta_{i}$ are near by degenerate and only two out of three can realistically be fitted for.

Issues related to the temperature dependence of the $r_{i}$ are discussed in Section 6.2. 


\subsection{ISS Mirror, Polarizing Beamsplitter, and Cleanup Polarizer}

The polarizing beamsplitter is treated as a perfect polarizer. Since the beam is not telecentric, the angle of incidence onto the beamsplitter coating, and thus the direction of polarization selected, depends on the vertical field position. At the center of the field, the direction is vertical and it changes by \pm 0.0174 radians or $\pm 1.00^{\circ}$ at the edge of the image (CCD). In calmode the maximum angle is 0.059 radians or $3.39^{\circ}$. This variation is taken as given (an error will appear as an apparent vertical dependence of $\theta_{3}$ ).

The polarization state selected from the light exiting the third waveplate is also affected by the ISS fold mirror since the direction of polarization of the light picked up by the beamsplitter is not perfectly parallel to the axis around which the mirror rotates the direction of the beam. However, this effect is very small and has not been included in the model.

Since the beamsplitter is assumed perfect, the cleanup polarizer should have no effect from a polarimetric point of view. However, it does cause a small amount $(<0.1 \%)$ of light to be lost due to the varying direction of the output from the beamsplitter.

\section{Test Description and Analysis Procedure}

The main calibrations were done using a couple of standard sequences. These were run during dedicated calibration times as well as during the Comprehensive Performance Tests, which were run several times to verify that the instrument survived transport and various environmental tests unchanged. These tests are described in Section 5.1, which also describes the way in which the overall coordinate system is determined. Tests for focus-block birefringence are described in Section 5.2 and those used for establishing the value of the degenerate combination of angular zero points in Section 5.3.

\subsection{Standard Tests}

These test sequences were initially constructed by combining a number of shorter sequences, each designed to measure the properties of one element assuming that the properties of the others are known.

A Singular Value Decomposition of the sensitivity matrix (Hessian) was then performed to determine if there were any degeneracies and if additional observations could be added to break those. This analysis revealed two issues. The first is that regardless of which observations are added, one linear combination of parameters is degenerate: The zero points $\theta_{1}, \theta_{2}$, and $\theta_{3}$ cannot be independently determined, as discussed in Sections 5.3 and 6.3.

The other issue is that some parameters are degenerate unless sequences moving more than one waveplate at a time are included.

Finally a number of redundant observations were removed.

In the end, the sequences used consist of these parts:

- Polarization sequences to determine the input polarization

- Sequences to determine properties of PCU polarizer

- Sequences to determine properties of PCU waveplate

- Sequences rotating each waveplate separately

- Sequences moving all waveplates together

In addition, observations were added between each block to track the stability of the light source. All observations also include darks to be able to account for temperature drifts in air with a high dark current (see Section 5.4.2). 
Table 1 List of polarization sequences used. $N$ indicates the number of good images from the front and side cameras. Type gives long (L) and short (S) sequence, as well as obsmode (O) and calmode (C). Roll is the PCU roll angle. It is left blank when unknown. Tests with FSN below 1000000 were performed at LMSAL. Tests with FSN between 1000000 and 1941258 were at GSFC. Tests after that as A. For definitions of the various sequences, etc., please see the following sections and Appendix B.

\begin{tabular}{|c|c|c|c|c|c|c|}
\hline FSN & $N$ & Type & Roll $\left({ }^{\circ}\right)$ & Box (C) & Window (C) & $\operatorname{Leg}(\mathrm{C})$ \\
\hline 440560 & $262 / 262$ & LO & & 24.34 & 25.23 & 21.28 \\
\hline 441084 & $140 / 140$ & $\mathrm{SO}$ & & 24.35 & 25.02 & 21.29 \\
\hline 441418 & $262 / 262$ & $\mathrm{LC}$ & & 24.34 & 25.19 & 21.23 \\
\hline 449822 & $140 / 140$ & $\mathrm{SC}$ & & 20.83 & 20.25 & 20.39 \\
\hline 450102 & $140 / 140$ & SO & & 20.81 & 20.16 & 20.31 \\
\hline 563654 & $140 / 140$ & $\mathrm{SO}$ & & 25.99 & 26.01 & 20.93 \\
\hline 563934 & $262 / 262$ & LO & & 26.09 & 26.06 & 21.11 \\
\hline 564466 & $140 / 140$ & $\mathrm{SC}$ & & 26.20 & 26.09 & 21.26 \\
\hline 878140 & $140 / 140$ & $\mathrm{SC}$ & 0.02 & 23.02 & 19.66 & 20.06 \\
\hline 879596 & $140 / 140$ & SO & 0.02 & 22.82 & 19.42 & 19.83 \\
\hline 947806 & $140 / 140$ & $\mathrm{SO}$ & 0.01 & 24.91 & 25.15 & 19.62 \\
\hline 963389 & $140 / 140$ & $\mathrm{SC}$ & 0.01 & 19.09 & 35.01 & 18.77 \\
\hline 963689 & $140 / 140$ & $\mathrm{SO}$ & 0.01 & 19.23 & 35.17 & 19.06 \\
\hline 1007964 & $140 / 140$ & $\mathrm{SO}$ & 90.17 & 25.25 & 21.45 & 21.25 \\
\hline 1010487 & $140 / 140$ & $\mathrm{SC}$ & 90.17 & 24.88 & 21.51 & 21.72 \\
\hline 1011869 & $140 / 140$ & $\mathrm{SC}$ & 90.17 & 25.78 & 30.95 & 21.66 \\
\hline 1012311 & $140 / 140$ & $\mathrm{SC}$ & 90.17 & 25.81 & 35.19 & 21.56 \\
\hline 1012679 & $139 / 139$ & $\mathrm{SC}$ & 90.17 & 25.80 & 38.95 & 21.51 \\
\hline 1019605 & $140 / 140$ & SO & 90.17 & 25.77 & 25.12 & 21.36 \\
\hline 1020025 & $140 / 140$ & $\mathrm{SC}$ & 90.17 & 25.62 & 24.91 & 21.33 \\
\hline 1230144 & $140 / 140$ & $\mathrm{SC}$ & 89.55 & 24.68 & 18.96 & 19.50 \\
\hline 1230432 & $140 / 140$ & SO & 89.55 & 24.53 & 19.13 & 19.67 \\
\hline 1765703 & $140 / 140$ & $\mathrm{SO}$ & 90.47 & 24.51 & 19.88 & 20.35 \\
\hline 1765983 & $140 / 140$ & $\mathrm{SC}$ & 90.47 & 25.10 & 20.54 & 20.76 \\
\hline 1955567 & $140 / 140$ & $\mathrm{SC}$ & 89.56 & 26.67 & 24.17 & 24.90 \\
\hline 1956735 & $140 / 140$ & $\mathrm{SO}$ & 89.56 & 26.94 & 24.46 & 25.32 \\
\hline 1988033 & $140 / 140$ & SO & 89.56 & 27.03 & 32.21 & 24.85 \\
\hline 1991769 & $140 / 140$ & SO & 89.56 & 26.96 & 24.05 & 24.88 \\
\hline
\end{tabular}

Appendix B summarizes the standard sequences used, while Table 1 gives a list of runs of the various sequences including temperatures etc. for future reference.

\subsubsection{PCU-HMI Roll Determination}

In order to get the zero point for the $(Q, U)$ coordinate system, it is necessary to determine the roll angle between the instrument and the PCU. This is done by moving an aperture stop in the PCU horizontally using the linear stage and taking images in calmode. The direction of motion of the image of this stop on the CCD gives the PCU-HMI roll. The rotation angles of the PCU polarizer and retarder are then corrected by this amount. A consequence of this is that operationally, horizontal is defined as the direction mapped into the row direction on the CCDs. 
This test sequence was run near the time of the calibration sequences taken beginning with Filtergram Sequence Number (FSN) 678221.

\subsection{Focus Block Tests}

Since the instrument is focused by inserting fixed-thickness focus blocks in the light path between the polarization selectors and the ISS beamsplitter, it is essential to determine if these blocks have any birefringence or cause other polarization artifacts. To this end the PCU is commanded to go through (approximately) $I+Q, I+U$, and $I+V$, with the instrument, at each, taking a polarization sequence with none and each of the six focus blocks individually in the beam.

\subsection{Determination of Degenerate Angle}

As mentioned in Section 5.1, $\theta_{1}, \theta_{2}$, and $\theta_{3}$ are degenerate and it is only possible to determine two distinct linear combinations. There are several possible solutions to this problem. One would be to decide that since the numbers are degenerate anyway one can pick an arbitrary $\theta$ for one of the motors, such as the numbers from Section 2.3, but as described those are not very accurate. Since there may be high-order effects (such as the incidence-angle effect mentioned in Section 4.4) depending on the actual angles this option is unsatisfactory. It is also possible that some high-order effect or the effects mentioned in Section 4.4 could be exploited but this was not pursued.

In the end it was decided to perform a one-time, special test taking four sequences:

- A standard polarization sequence with all selectors present

- A sequence with all polarization selectors removed

- A sequence with only the first selector present

- Another standard sequence with all selectors present

Note that the polarization selectors were taken in and out by hand, which means that there may have been small alignment changes.

\subsection{Data Analysis Procedure}

The data analysis consists of several steps:

\subsubsection{Bad Image Rejection}

First any incomplete images caused by problems with the data collection are detected and rejected. As shown in Table 1, no more than one image per camera had to be rejected. Given the redundancy in the sequences this is not a significant problem.

\subsubsection{Dark Subtraction}

For observations in air, the CCDs are at room temperature and the dark current is thus large, at times exceeding the signal. Furthermore the temperature (and thus dark current) often changes during the time of the observations and images taken after a long pause (e.g. after moving PCU elements) often show slightly different dark levels. For these reasons two darks are taken at the beginning of each sequence of images of which the first is discarded and the second is used to make a model of the dark level as a function of time, which is then subtracted from the other images.

A rough flat field was also applied, although this has little effect on other parameters than those describing the light source. 


\subsubsection{Binning}

Since individual pixels are noisy and since the spatial dependencies of the parameters are gradual, the images are binned to either $1 \times 1$ and $32 \times 32$ for the results presented here.

\subsubsection{Fitting}

Although it makes little physical sense (since many elements are rotating), each pixel is fitted separately. Given an initial guess for each of the parameters and the state of each mechanism, the model intensity and its derivatives with respect to each of the parameters are calculated. The best fit is then found using a non-linear minimization procedure.

\section{Results}

The number of results obtained from the numerous calibrations is very large, and thus only the main results are reported below. Except for determining temperature dependencies, results are typically only shown for one run of the sequences and only for the front camera. The other runs, including those for the side camera were analyzed and the instrumental results were found to be very similar.

\subsection{Parameter Maps}

Figures 3 through 8 show maps of the various fitted parameters near the planned operating temperature of $20^{\circ} \mathrm{C}$. Unless otherwise noted obsmode results use the series at $\mathrm{FSN}=450102$, while calmode results are for the series at $\mathrm{FSN}=449822$.

\subsubsection{Stimulus Telescope Parameters}

Figure 3 shows variations of the order of $10 \%$ in the obsmode intensity, due to imperfections in the light feed, while the calmode intensity is more uniform. The patterns show significant variations, likely from differences in alignment during setup for the different runs.

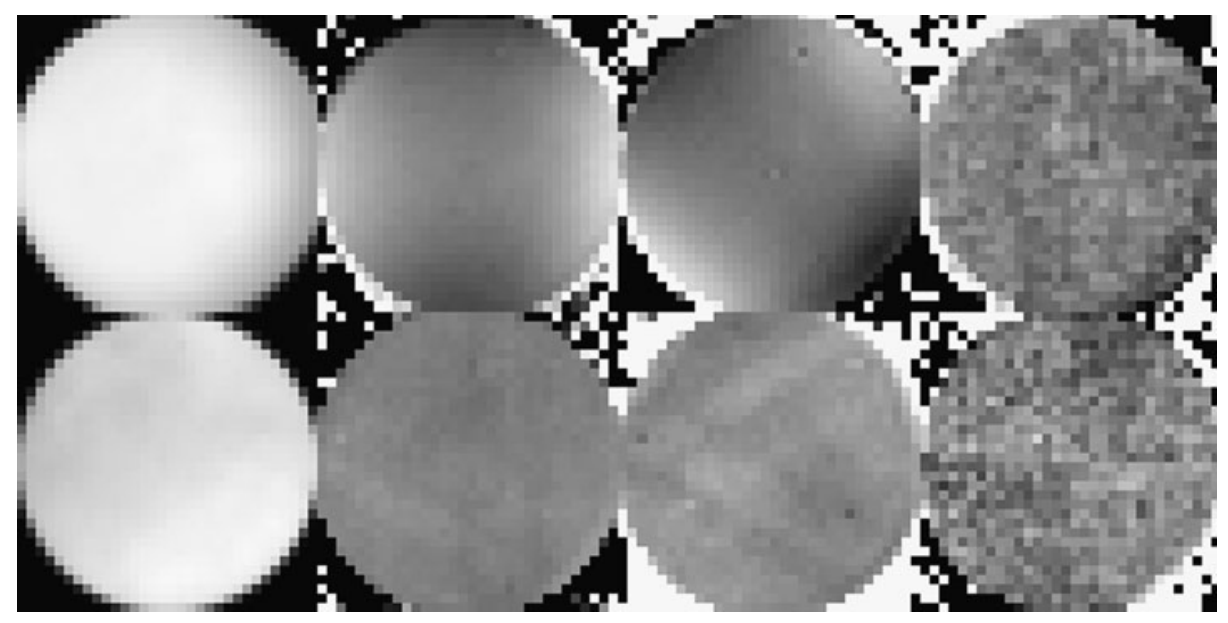

Figure 3 Parameters for the stimulus telescope light. Front left to right: Intensity (0 to maximum), $Q / I$ $( \pm 0.01), U / I( \pm 0.01)$ and $V / I( \pm 0.001)$. The top shows obsmode, the bottom calmode. 
The input polarization in obsmode shows an axisymmetric linear-polarization pattern with an amplitude around $0.5 \%$, likely caused by the angle of incidence on some optics having a strong field dependence combined with an imperfect anti-reflective (AR) coating. The likely culprit is the condenser lens in the stimulus telescope, which is very close to focus and has a small radius of curvature. There is little input circular polarization. The polarization is very small in calmode, where the obsmode numbers have, effectively, been spatially averaged.

\subsubsection{PCU Parameters}

Figure 4 shows $I_{x}$ and $I_{y}$ from Equation (6), which describe the effect of the image offsets caused by the two PCU elements. As expected the patterns are roughly proportional to the corresponding derivatives of $I$. There are significant outliers in these plots, caused by dust specks and scratches on the condenser lens and, as expected, part of the pattern rotated by $90^{\circ}$ as the instrument was rotated relative to the stimulus telescope from the Lockheed Martin Solar and Astrophysics Laboratory (LMSAL) to the Goddard Space Flight Center (GSFC)/Astrotech Science Operations (ASO) configuration (see Table 1). Unfortunately the linearization is not perfect and there are thus artifacts in some of the other parameters.

The remaining PCU parameters are shown in Figure 5. The polarizer transmits about $88.9 \%$ of the desired polarization, with a standard deviation of around $0.1 \%$. For unknown

Figure 4 PCU offset parameters (see Equation (6)). Left panel shows $I_{x}$, right panel $I_{y}$. Scale is arbitrary.
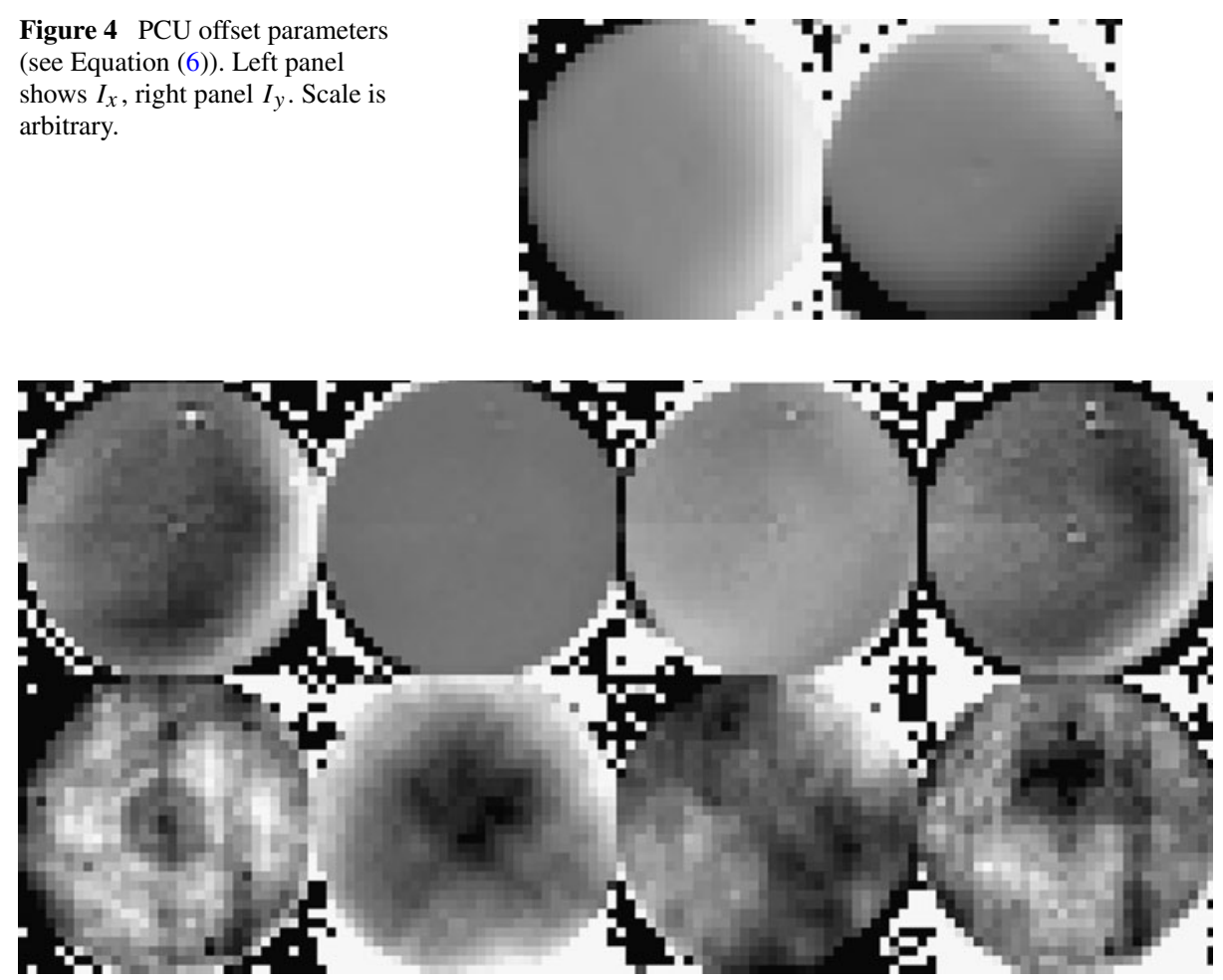

Figure 5 PCU element parameters. From left to right: Polarizer transmission (scale from 0.886 to 0.891), retarder retardance $(0.209$ to 0.214$)$, retarder angle $(-0.011$ to -0.003 radians) and retarder transmission ( 0.990 to 0.997$)$. Obsmode is in the top row, calmode in the bottom. 
reasons the transmission was derived to be higher by about $0.5 \%$ for two of the runs. The parameters show little variation in obsmode (where any non uniformities are averaged out). In calmode (where the elements are near focus) there is a clear radial dependence for some of the parameters. The retarder angle shows some variations, but nothing systematic from run to run. The mean of -0.0055 radians or $-0.3^{\circ}$ is roughly consistent with the error estimate of $0.2^{\circ}$.

The PCU parameters appear to be consistent between obsmode and calmode when averaged spatially.

\subsubsection{Window Parameters}

Two types of parameters describe the HMI front window: one giving the amount of depolarization, the other the birefringence. As described in Section 4.3 a six parameter model is normally used, although a five-parameter model with explicit depolarization and birefringence parameters was also implemented. Figure 6 shows the result of such a fit (which at nominal operating temperature is as good as the six-parameter fit). The first thing to note is that the depolarization is very low. In obsmode the averages of $D_{Q}, D_{U}$, and $D_{V}$ (see Equation (9) in Appendix A.2) are within $0.25 \%$ of 1 , with $D_{Q}$ slightly larger than 1 , likely due to a small systematic error of unknown origin. The total birefringence is less than $0.1 \%$ of a wave.

The calmode results are more interesting. The birefringence, while small, shows a triangular pattern, likely due to a small amount of stress induced by the three-point mountings of the front window and primary lens. During the early part of the integration a test $(\mathrm{FSN}=183462)$ was run without the window installed and the maps show that several of the features are still present, implicating the primary lens for at least some of the effects.

The effects of the window parameters are temperature dependent, as discussed in Sections 6.2.2 and 7.2.2.

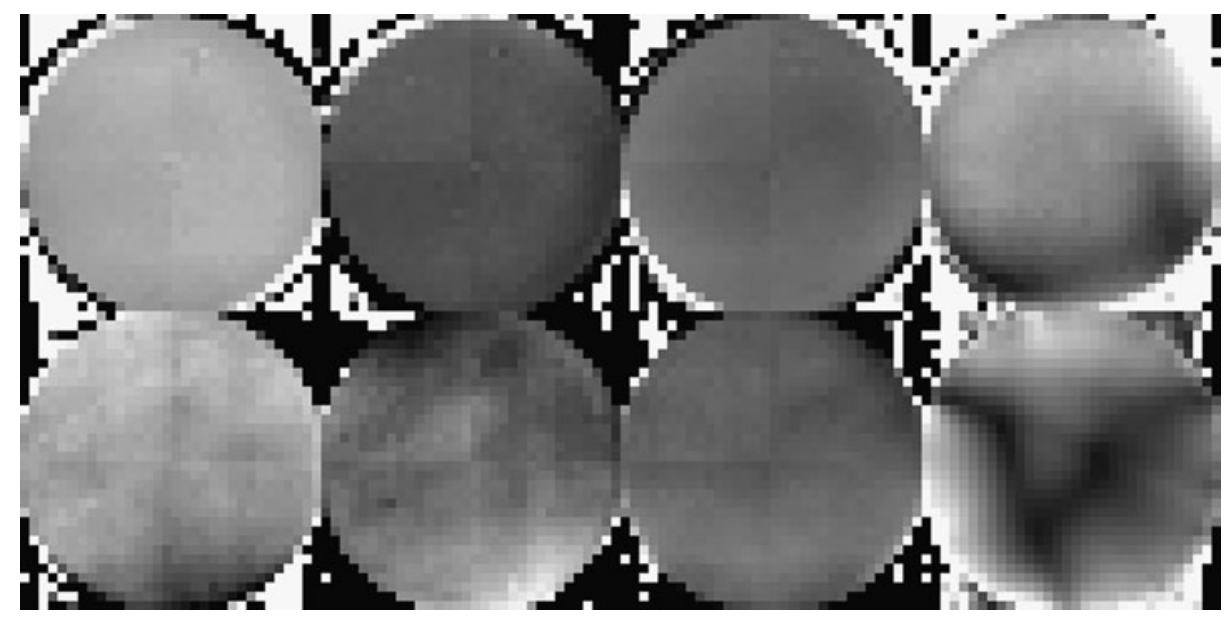

Figure 6 Front-window parameters in obsmode (top) and calmode (bottom). Three left columns show the depolarization terms on a scale from 0.99 to 1.01 (see text). The right-hand plots show the total birefringence $\sqrt{M_{Q V}^{2}+M_{U V}^{2}}$ on a scale from 0 to 0.01 ( 0 to 0.0016 waves) in obsmode and 0 to 0.15 ( 0 to 0.024 waves) in calmode. 


\subsubsection{Polarization Selector Parameters}

The waveplate retardances $\left[r_{i}\right]$ and zero points $\left[\theta_{i}\right]$ are shown in Figures 7 and 8 , respectively. The average for PS1 corresponds to a zero point in steps of 63 and the value for PS3 to 9, in good agreement with the estimates given in Section 2.3.

All parameters show some variations, most of which are consistent from test to test, but not between obsmode and calmode. Since the waveplates rotate between measurements, the spatial variations clearly cannot represent the true variations and at best represent averages. In any case the variations are small and not a cause of concern.

The value of $\theta_{3}$ is degenerate with the angle selected by the polarizing beamsplitter. If this angle varies spatially due to unmodeled properties of the beamsplitter or ISS mirror, this will appear as an error in $\theta_{3}$. Similarly, it is assumed that the illumination is independent of

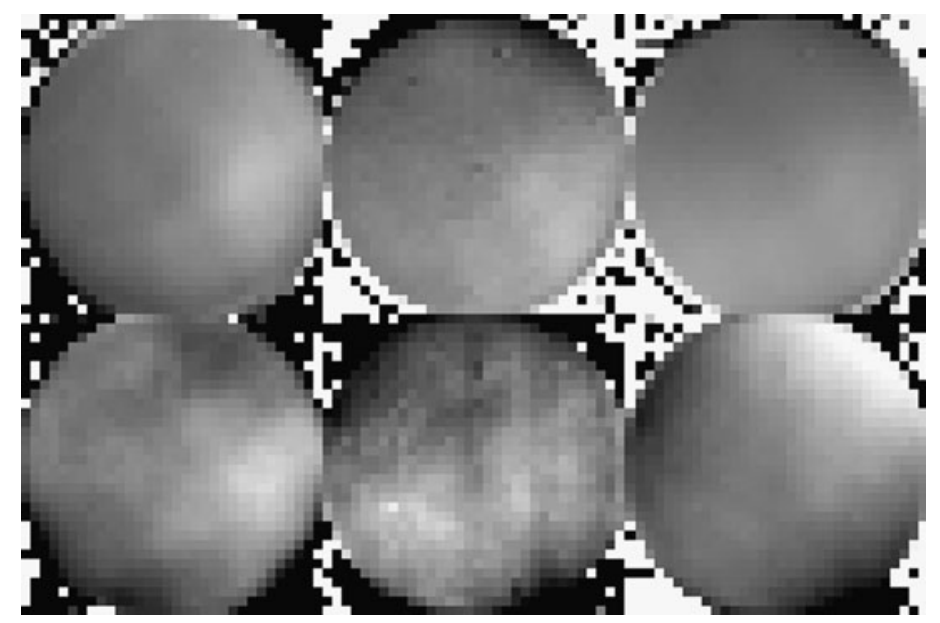

Figure 7 Retardances minus averages in obsmode (top) and calmode (bottom). From left to right are PS1 (at \pm 0.001 waves), PS2 (at \pm 0.001 waves), and PS3 (at \pm 0.0025 waves). Averages and standard deviations in obsmode are 10.5000 $\pm 0.0002,10.2520 \pm 0.0003$, and 10.4981 \pm 0.0005 . In calmode $10.4999 \pm 0.0003$, $10.2517 \pm 0.0003$, and $10.4980 \pm 0.0010$.

Figure 8 Retarder zero points $\theta_{1}$ (left) and $\theta_{3}$ (right) in obsmode (top at \pm 0.001 radians relative to mean) and calmode (bottom at \pm 0.005 radians relative to mean). Temperature for this measurement was $20.8^{\circ} \mathrm{C}$. Averages and standard deviations are $-1.6559 \pm 0.0002,-2.3797$

(see Section 6.3) and $0.2408 \pm 0.0006$ radians in obsmode and $-1.6556 \pm 0.0008$, -2.3797 (see Section 6.3) and $0.2399 \pm 0.0016$ in calmode.

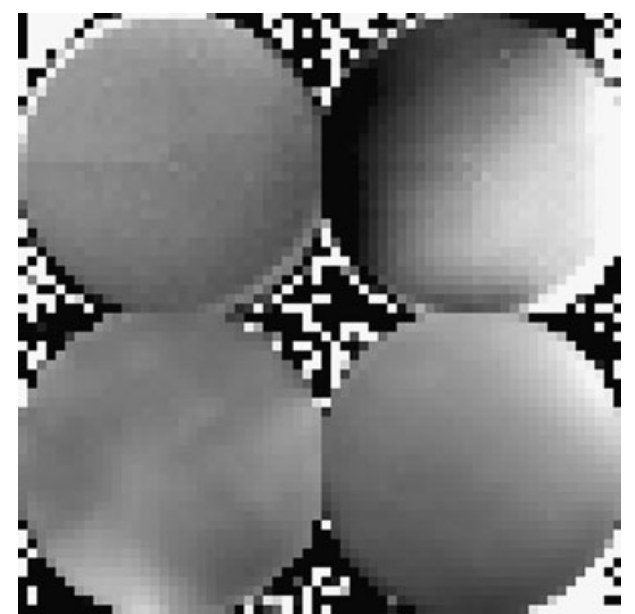


pupil position in obsmode and of the field position in calmode and that the average angle selected is thus, to first order, that of the central ray. However, the illumination is not totally uniform and varies from test to test, which may explain some of the temporal variations seen.

\subsubsection{Quality of Fit}

One of the ways in which one can assess the overall accuracy of the calibration is by examining the residuals. Figure 9 shows the quality of the fits for integrated light. The rms deviation is of the order $0.44 \%$ of the mean intensity.

A possible cause of residuals is the lamp intensity. To test for this, a sequence taking two darks, 50 identical images, and a dark on both cameras, all at the same cadence as used for the regular observations, was taken before each test. The resulting variations range from better than $100 \mathrm{ppm}$ to somewhat over $1000 \mathrm{ppm}$. Larger values generally indicate that the bulb is about to fail or has not been adequately warmed up. In those cases the bulb is replaced and/or the lamp is warmed up and the test repeated. In any case the variations fail to explain a significant fraction of the residuals observed. Figure 9 shows a linear temporal trend in the residuals, likely due to the lamp changing intensity. However, this change is small, and correcting for it does not improve the fit significantly.

This is consistent with the residuals being correlated from test to test in the same configuration. The residuals between obsmode and calmode are significantly correlated in the same configuration. When the configuration changed from LMSAL to GSFC/ASO (causing the instrument to rotate $90^{\circ}$ relative to the stimulus telescope) the residuals are almost uncorrelated.

Figure 10 shows the rms residuals as a function of position. In un-averaged light the rms over the images is $0.38 \%$ in obsmode and $0.69 \%$ in calmode, both with significant

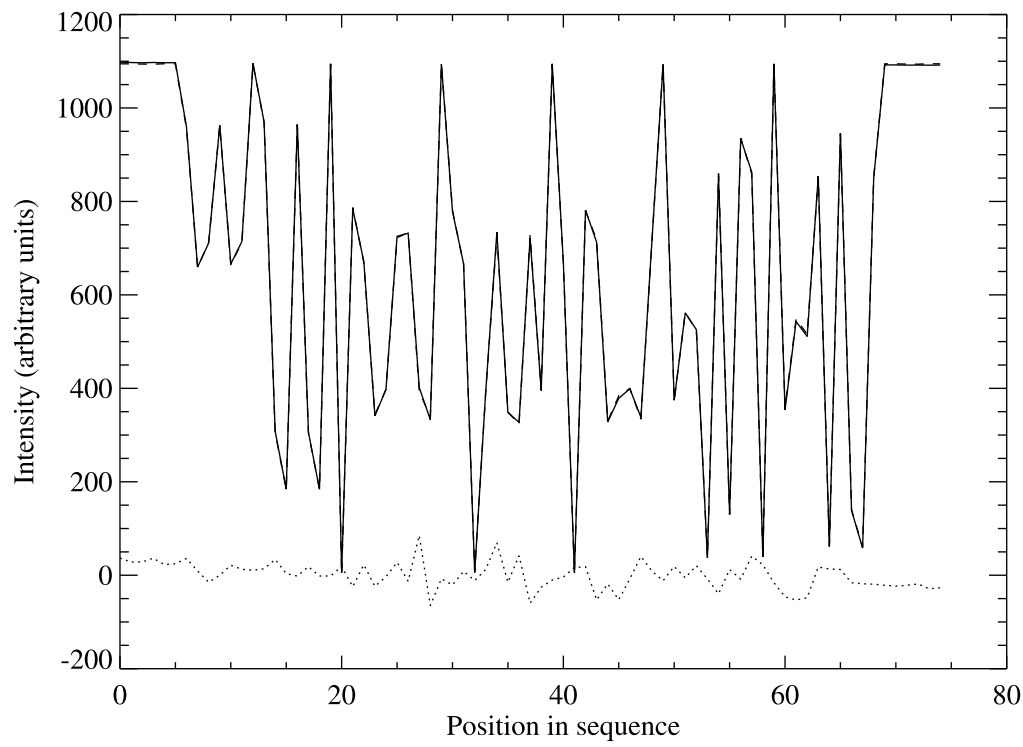

Figure 9 Spatially averaged intensity (solid line) and fit (dashed line) as a function of position in a standard (short) test sequence. Darks have been excluded. Note that the dashed line is barely visible indicating an excellent fit. This is further illustrated by the dotted line which shows ten times the residuals. 
Figure 10 Rms residuals, divided, at each point, by the mean intensity at that point. Obsmode (left from 0 to $0.6 \%$ ) and calmode (right from 0 to $1.6 \%$ ).
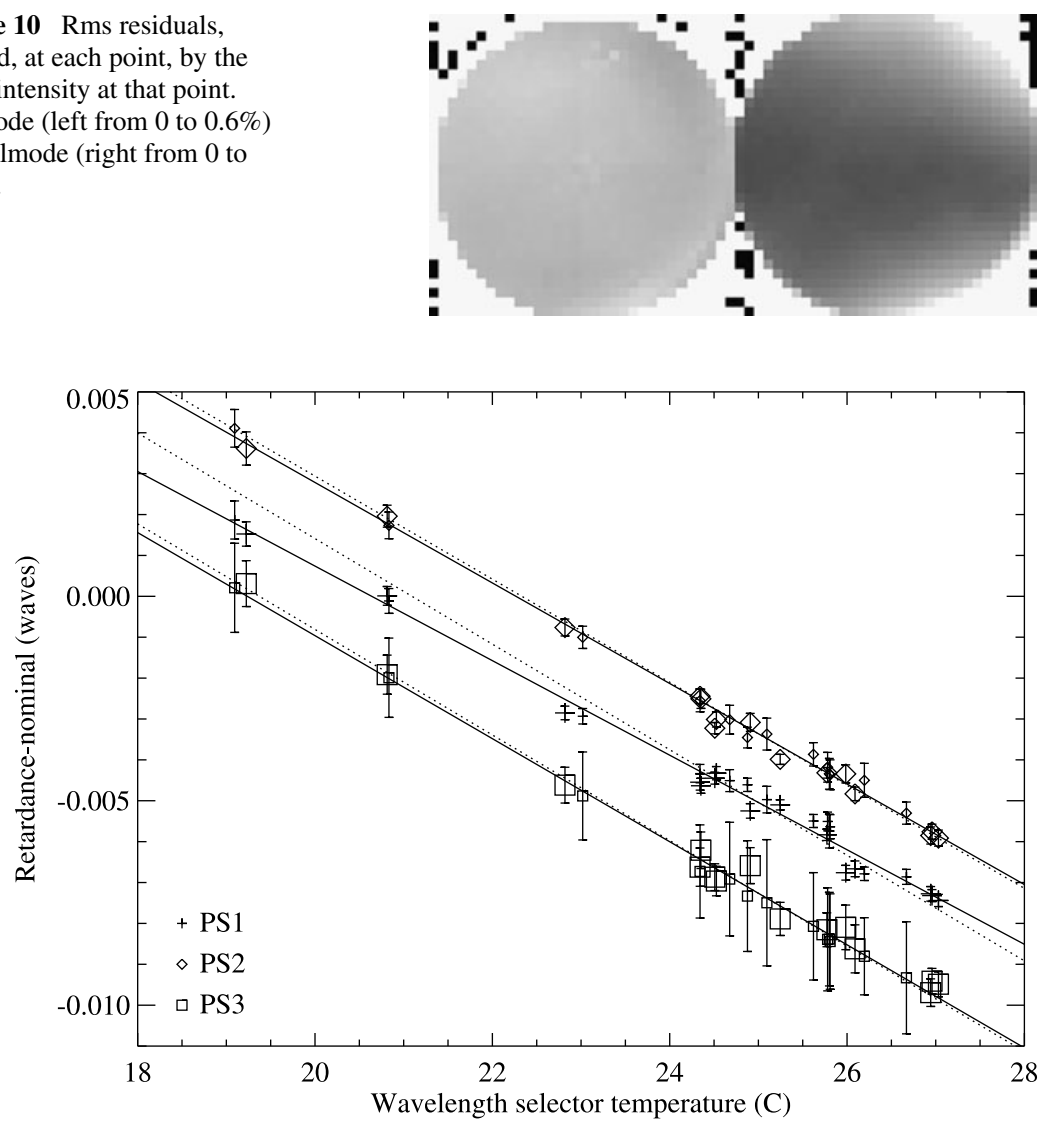

Figure 11 Waveplate retardances as a function of temperature. The values were averaged over $r \leq 0.95$ and the nominal values $(10.5,10.25$, and 10.5 waves) subtracted. Error bars show the standard deviation over the averaging area. Large symbols are obsmode, small symbols calmode. Solid lines are fits. The slopes are -0.00116 waves $\mathrm{C}^{-1},-0.00123$ waves $\mathrm{C}^{-1}$, and -0.00126 waves $\mathrm{C}^{-1}$ for PS1, PS2, and PS3, respectively. Dotted lines show the values assuming a fractional change of $-1.23 \times 10^{-4} \mathrm{C}^{-1}$ relative to the numbers at $25^{\circ} \mathrm{C}$.

variation. The obsmode residuals are fairly uniform while the calmode residuals have a very systematic pattern, likely due to some neglected physical effect, possible the same one causing the systematic residuals mentioned above.

\subsection{Temperature Dependence}

In principle, many components vary with temperature. However, we found only two significant temperature effects on the polarization properties: the waveplate retardances and front-window birefringence.

\subsubsection{Waveplate Temperature Dependence}

Figure 11 shows that the retardance variations are close to linear and to that expected from a fractional change of $-1.23 \times 10^{-4} \mathrm{C}^{-1}$ (Etzel, Rose, and Wang, 2000). The rms deviations 
of the individual means from the fit are of the order of 0.0002 waves, meaning that it is straightforward to model them with the required accuracy.

The retardances are essentially independent of the configuration, in other words the values are the same in obsmode and calmode and in air and vacuum (not shown).

The residuals from the fit could be due to temperature gradients inside the instrument. The temperature used for Figure 11 is that of a sensor on the motor mount, and there could be a systematic difference between that and the actual optics.

When the front window is heated to create a large temperature gradient (see the next section), $\theta_{1}$ shows large spatial variations in calmode. This is likely to be spurious due to the window model being inadequate in that case and is of little consequence since there are no plans to operate with a large gradient.

\subsubsection{Front Window Birefringence}

Maps of $M_{Q U}, M_{Q V}$, and $M_{U V}$ (see Appendix A.4) at selected environment-to-window temperature differences are shown in Figure 12. Clearly there is significant stress birefringence, which could be acquired in at least two ways.

Bulk temperature change. As described in Section 2.1 the front window is a sandwich of different materials with different temperature-expansion coefficients that will induce stress in each other when the temperature is different from some reference value (presumably close to the assembly temperature).

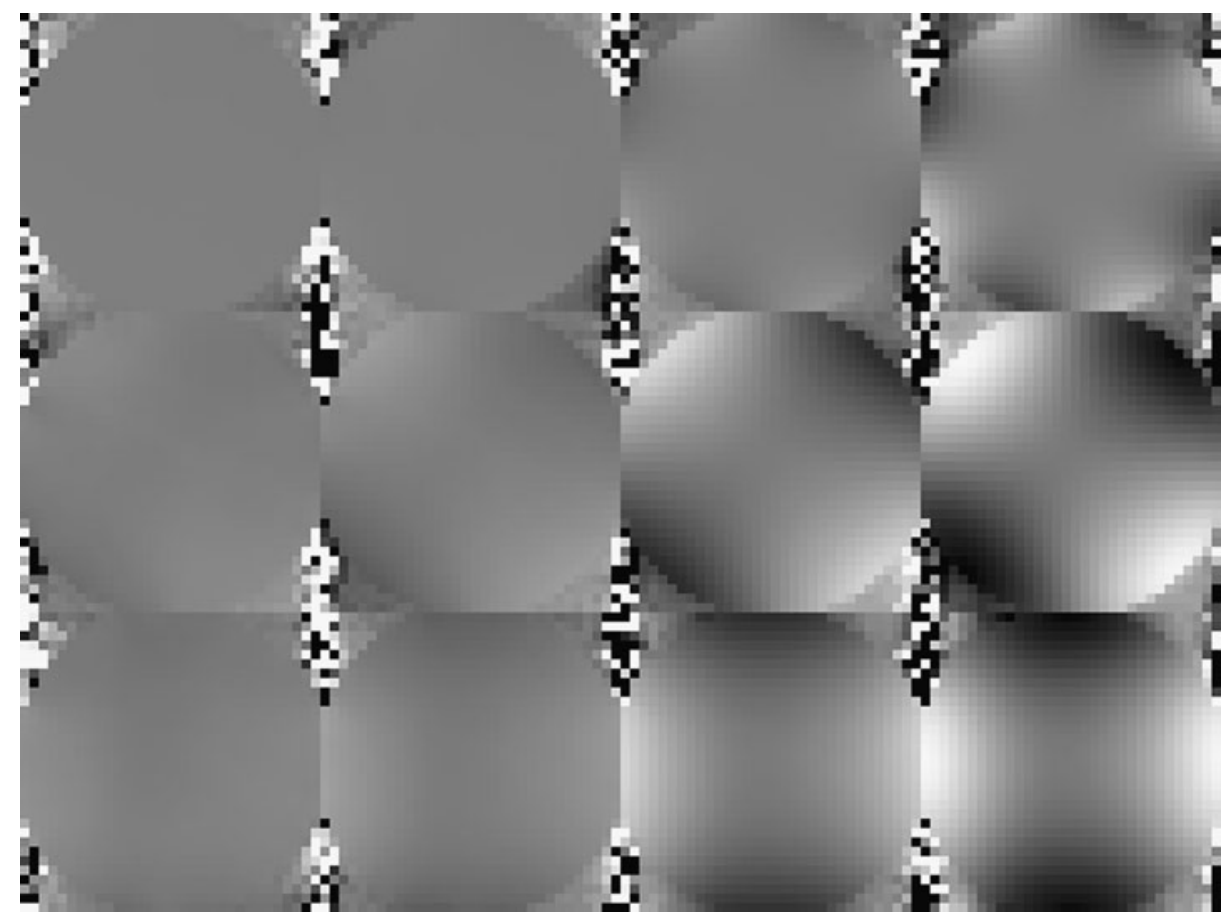

Figure 12 Window parameters in calmode: $M_{Q U}$ (top row), $M_{Q V}$ (middle row), and $M_{U V}$ (bottom row) for four values of the leg-to-window temperature difference: $-0.1^{\circ} \mathrm{C}, 4.8^{\circ} \mathrm{C}, 9.3^{\circ} \mathrm{C}$, and $17.4^{\circ} \mathrm{C}$. Scale is from -1 to 1 . 
Temperature gradients. Since the edge and center of the window are coupled differently to the environment, a radial temperature gradient can develop. In air one may reasonably assume that the dominant term determining the gradient is the difference between the air temperature and the temperature of the rim of the window, perhaps coupled with the amount of air flow. In vacuum, the gradient is presumably determined by a competition between the heat applied by the rim heater, on the one hand, and radiation to the environment and the interior, on the other. In either case the expected temperature distribution is, to lowest order, given by a cosh function of radius, which over some parameter range may be approximated by a quadratic.

This effect, in principle, also applies to other optical elements, but those are internal to the box, and see a well-regulated and homogeneous thermal environment. A likely exception to this is the primary lens, which is coupled closely to the window but which is in any case treated together with the window.

Determining which effect is dominant requires modeling or tests in different environments. Unfortunately it was not possible to test at a grid of air and rim temperature combinations (see Figure 13), and so it is not possible to do a clean analysis.

Assuming that the stress birefringence is small and linear in the temperature (or temperature difference), it is possible to correlate $M_{Q V}$ and $M_{U V}$ against the relevant temperatures. The result is that $M_{Q V}$ and $M_{U V}$ have correlation coefficients of $96.7 \%$ and $96.9 \%$ against the front-window temperature and $98.7 \%$ and $98.8 \%$ against the difference between the environment temperature and that of the front window. This strongly points to the temperature gradient being the culprit. $M_{Q U}$ is quadratic in the birefringence to lowest order and was therefore not used for the correlation, but it is given quite accurately by Expression (10) in Appendix A. 3 from $M_{Q V}$ and $M_{U V}$.

Figure 14 shows that the birefringence temperature dependence is roughly quadratic with radius. Also shown is a fit of a cosh function which, as discussed earlier, is the expected

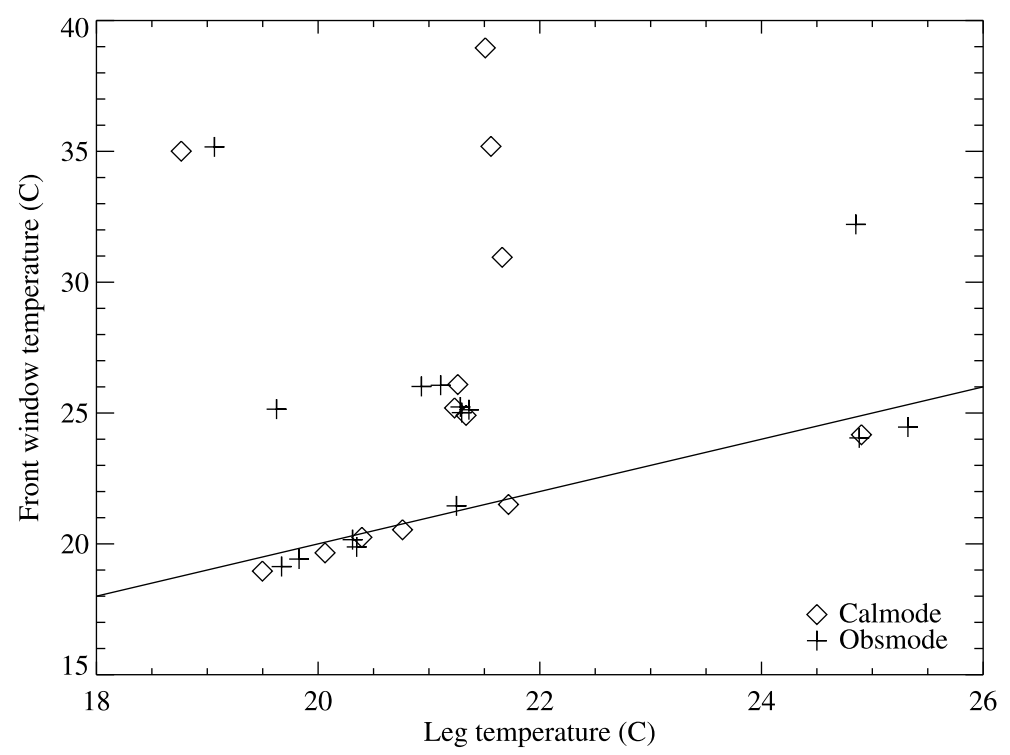

Figure 13 Available leg (environment) and front-window temperatures combinations. Straight line shows a one-to-one correspondence. Points below the line are likely due to the leg temperature not being a perfect representation of the environment and/or imperfect calibration of the temperature sensors. 


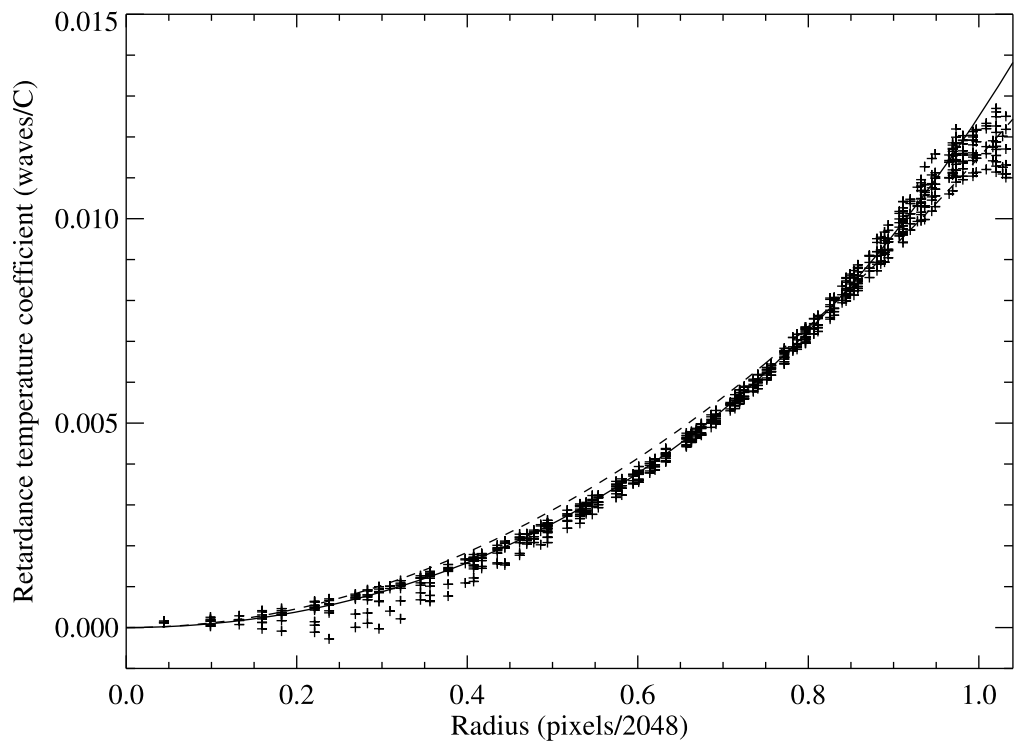

Figure 14 The temperature coefficient of the birefringence. The turnover near $r=1.0$ is likely caused by the birefringence being close to $1 / 4$ wave at the highest temperature causing the simple equation retardance $=\sin ^{-1}\left(\sqrt{M_{Q V}^{2}+M_{U V}^{2}}\right)$ to break down. Also shown is a quadratic $0.0115 r^{2}$ (dashed) and a fit of a cosh function $0.0125(\cosh (1.85 r)-1) /(\cosh (1.85)-1)($ solid $)$.

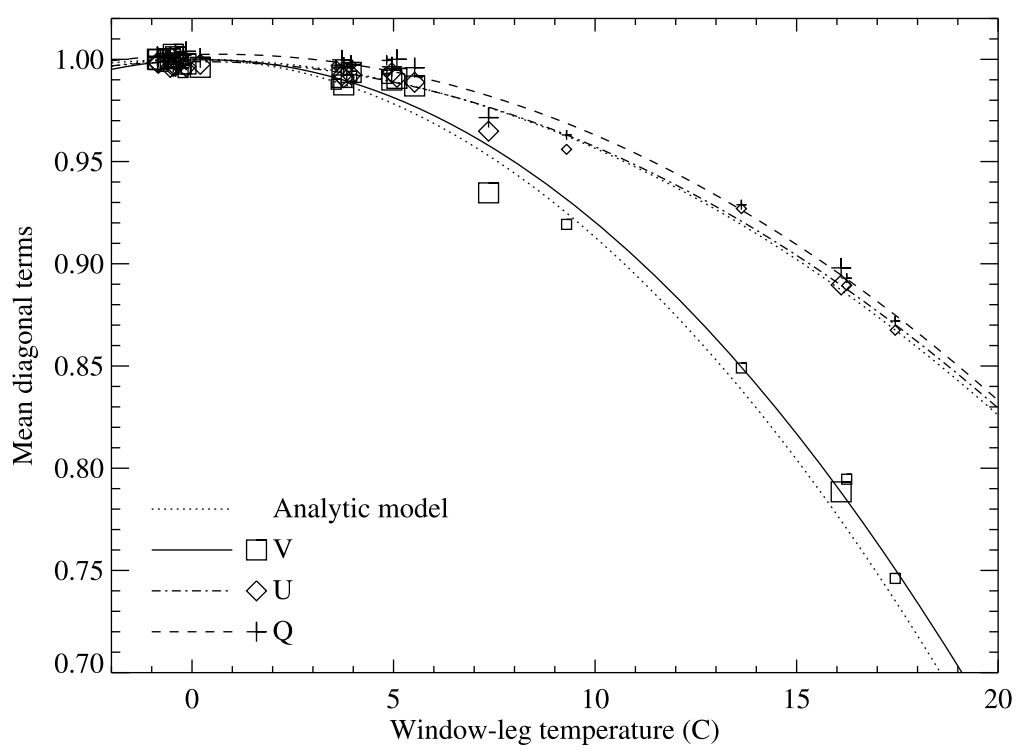

Figure 15 Temperature coefficient of the diagonal terms of the window Mueller matrix. Large symbols show obsmode and small symbols calmode. The dotted lines shows the values obtained from the fit in Figure 14 expanding the Mueller matrix of a waveplate to second order in the retardance and integrating the result over the aperture. Solid, dashed, and dash-dotted lines show quadratic fits. 
functional form of the radial temperature dependence. The minimum birefringence (in an rms sense) is reached for a temperature difference of $-1.5^{\circ} \mathrm{C}$, that is when the window rim is colder than the environment. However, the proxy for the environment temperature (that of a mounting leg) is not necessarily an accurate representation, and so it is not clear that this is significantly different from zero.

Figure 15 shows the effect of a temperature gradient on the diagonal elements of the window Mueller matrix, which roughly gives the amount of depolarization. The numbers are reasonably well represented by the quadratic fits, as well as from the value expected from the quadratic relation shown in Figure 14. The maxima of the fitted curves are located between $0.2^{\circ} \mathrm{C}$ and $0.7^{\circ} \mathrm{C}$ with values between 0.999 and 1.0025 . In other words the depolarization is very small when the window is at a uniform temperature. The deviations from the fit may be caused by the difference between the true environment and that measured by the leg temperature, and by the fact that the temperatures sometimes vary by $1{ }^{\circ} \mathrm{C}$ or more during the observations.

It is clear that the window effects are significant and will require attention when constructing observables. A calibration to find the effective environmental temperature will have to be performed on orbit (see Section 7.3.2). A large amount of birefringence will not only result in depolarization (which may in principle be corrected for), it will also result in what effectively amounts to a polarization-dependent point-spread function.

Some implications of the temperature dependencies are discussed in Section 7.2.2.

\subsection{Determination of Degenerate Waveplate Rotation Angle}

The instrument was in an unusual configuration for this test with the Lyot filter removed, resulting in unusual light paths through parts of the instrument. For that reason only a single spatially averaged number (with unknown averaging) was determined.

The images were analyzed with the standard analysis code modified to allow for a fit of $\theta_{2}$ and for missing elements. The result was $\theta_{2}=-2.379742$ radians or $-136.349^{\circ}$, within the uncertainty of the value of $-133.5^{\circ}$ determined before assembly (see Section 2.3).

\subsection{PCU Roll Determination}

As mentioned in Section 5.1.1, measurements of the PCU to HMI roll were taken in connection with the main polarization measurements, starting with FSN 678221 . Occasionally the automated algorithm used yields a bad value, which has to be rejected, but otherwise the offsets fall on a straight line to within better than one pixel. Since the two cameras are not perfectly aligned, the angle derived is different, typically by around $0.083^{\circ}$ or 0.0015 radians, consistent with the value found by Wachter et al. (2010). For the calculations here, the mean angle is used for both cameras.

To test this procedure, a pair of tests (FSN 678221 and 678 361, which are not otherwise used) were performed. One with a normal setup, the other with one side of the PCU propped up by $12 \mathrm{~mm}$, which with a lever arm of about $800 \mathrm{~mm}$ should result in a tilt of 0.015 radians. The measured roll change was 0.0147 radians, well within the knowledge of the tip. After correcting the PCU angles by this number, $\theta_{1}$ changed by 0.00081 radians, indicating a very accurate correction.

For the series taken before FSN 678221 , a constant was added to $\theta_{1}$ to make the mean of each determination equal to the ones taken after FSN 678221. 


\subsection{Focus Blocks}

The images for each input polarization and focus block are demodulated using a simple polarization model. The case with no focus blocks is then subtracted from the cases with focus blocks in the beam, thereby canceling out other PCU and instrument defects to lowest order.

The results typically show spatially uniform differences from zero to $0.5 \%$ or so. However, there does not appear to be a persistent pattern to the changes and the effects seen are most likely due to the variable dark current mentioned in Section 5.4.2. Consistent with this, the spatial variations in polarization are significantly smaller, typically around $0.05 \%$ to $0.1 \%$.

\section{Discussion}

In the previous sections the polarimetric model was described and the results presented. This section starts with a discussion in Section 7.1 of the overall accuracy of the estimated parameters, followed in Section 7.2 by a discussion of the features that are currently planned to be in the polarimetric model used for the calibration of the science data. Finally, Section 7.3 describes the plans for updates to the calibration after launch.

\subsection{Accuracy of Derived Parameters}

It is difficult to reliably assess the errors in the various measured quantities, but there are a number of hints about how large they may be. Among these are the rms residuals versus settings and image position, the (unphysical) spatial variations of the waveplate parameters, the variations between runs, the residuals from the fits versus temperature (Section 6.2), and how these errors propagate into the error matrix (Section 7.2.3).

The reconstruction errors were shown in Figure 9 and discussed in Section 6.1.5. These probably represent a lower bound on the errors in the combined PCU-HMI system. Unfortunately, it has not proven possible to determine their origin, and so it is unclear if they are related to the PCU or the instrument.

The up-down variation in calmode residuals in Figure 10 likely indicates the neglect of some physical effect. That it does not appear in obsmode could indicate that it is related to the steep angles through the area around the polarization selectors. The angles have been taken into account for the polarization selectors and the polarizing beamsplitter, but a possible candidate is the ISS mirror, which is likely not perfect (e.g. the reflectivity and the phase delay on reflection are different for $\mathrm{s}$ and $\mathrm{p}$ polarization). However, since the effect is negligible in obsmode, this issue has not been pursued.

The spatial variations of the waveplate retardances and zero points are, as discussed in Section 6.1.4, very small and are in some cases degenerate with other (unfitted) parameters and may thus, to some extent, reduce the errors in the calibrated results.

\subsection{Resulting Polarimetric Model}

\subsubsection{High-Incidence-Angle Effects}

Figure 16 shows the inferred retardances with and without including the effect of the finite incidence angles on the polarization selectors. The effect is quite significant in calmode, 


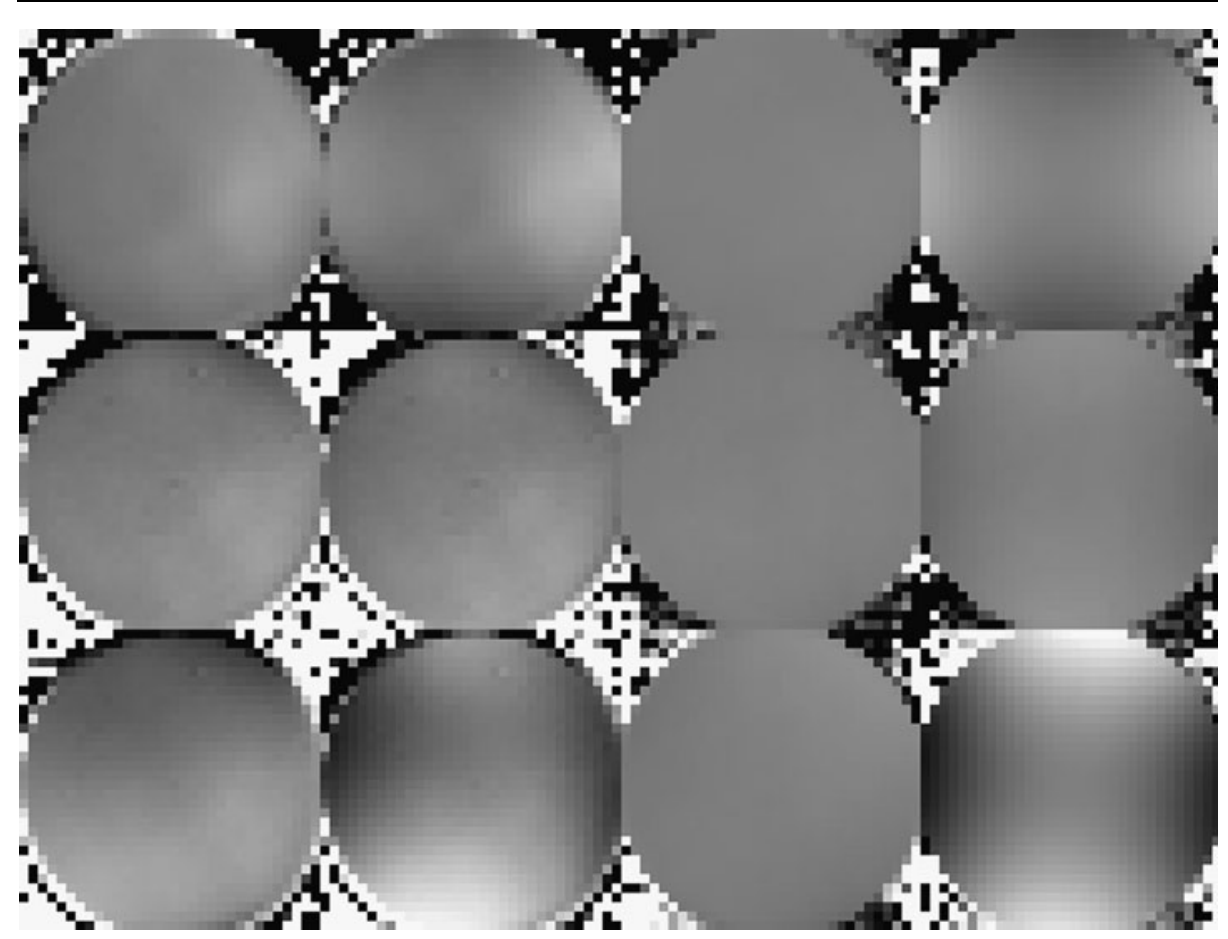

Figure 16 Waveplate retardances with and without correcting for the angle-of-incidence effect in the waveplates. Rows are the retardances of PS1 (top), PS2 (middle) and PS3 (bottom). Two left columns are in obsmode with and without the correction on a scale of \pm 0.002 waves. The two right columns the corresponding calmode results on a scale of \pm 0.02 waves.

especially for PS3, where including the effect makes the rms variation decrease from 0.0072 waves to 0.00097 waves. In obsmode the corresponding decrease is from 0.00072 to 0.00048 waves. The mean rms reconstruction error in calmode is reduced from $1.71 \%$ to $0.71 \%$, again indicating a significant effect. The obsmode improvement from $0.48 \%$ to $0.45 \%$ is more modest.

Given the magnitude of the improvements, it is clear that this effect should be included, even if there is no requirement to calibrate calmode data.

\subsubsection{Models of Spatial and Temperature Dependence}

To perform the demodulation described in Section 1.1 it is necessary to have a model that will reliably predict the model parameters given instrument settings and environmental conditions, such as the window and polarization-selector temperatures. The prescription described below represents the current plans and will be revisited after on-orbit data have been taken.

To obtain the spatial dependence, it is necessary to smooth over the minor defects in the parameters due to dust specks etc. and to extrapolate the data between the edge of the valid area and the edge of the FOV used. This is done by fitting a low (fifth) order polynomial in $x$ and $y$ to the data out to a fractional radius of 0.95 .

The window is modeled using the six-parameter model with the temperature effects for the $(Q, U, V)$ part of the matrix given by a quadratic in window-environment temperature 
Figure 17 The demodulation errors resulting from modulating using a set of derived parameters (FSN 450 102) and demodulating using the model described in Section 7.2 for the corresponding temperatures. Columns have the different input polarizations, rows the outputs. Each sub image shows a spatial map of the corresponding element of the matrix $\mathcal{E}=\mathcal{D} \mathcal{O}-\mathcal{I}$ (see Section 1.1). Scale is $\pm 1 \%$.

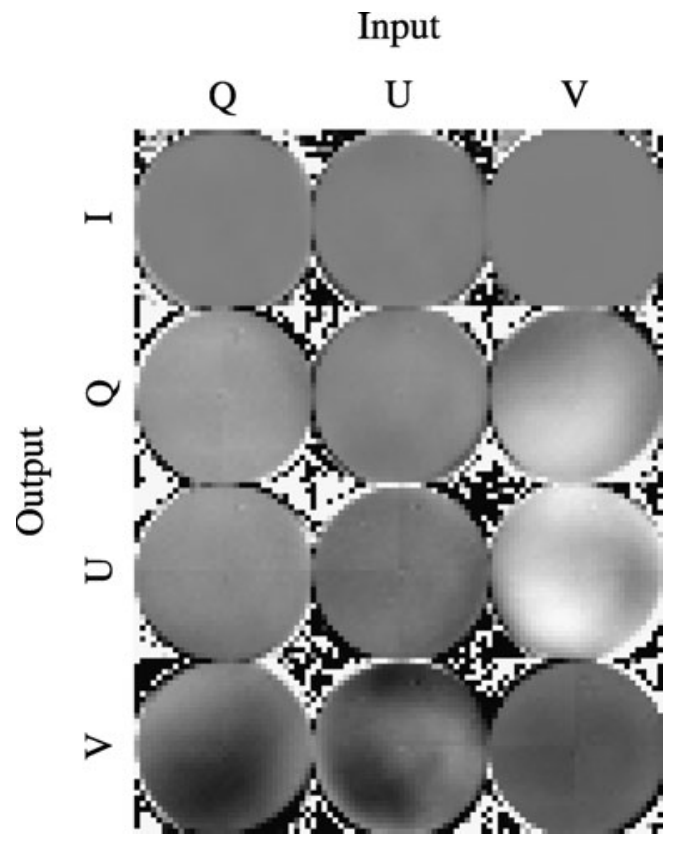

for the diagonal (depolarization) terms and by a constant for the off-diagonal terms. The determination of any window effects related to inherent polarization has been deferred to on-orbit, as has the determination of the effective on-orbit environment temperature.

The retardances of the polarization selectors will be modeled by the fits shown in Figure 11. The zero points will be assumed to be constant.

\subsubsection{Demodulation Accuracy}

Given the polarimetric model mentioned above, it is possible to calculate the expected parameters and the demodulation matrix $\mathcal{D}$ (obtained by a least-squares method from $\mathcal{O}$ ) for each calibration run, similar to what would be done for on-orbit data. For a given run and a given observing sequence, one can similarly calculate the modulation matrix $\mathcal{O}$ from the actually determined parameters and demodulate it using $\mathcal{D}$, thereby getting an estimate of the error matrix $\mathcal{E}=\mathcal{D} \mathcal{O}_{\text {true }}-\mathcal{I}$. Figure 17 shows a representative result.

Excluding the calibration sequences for which the window was excessively hot the rms deviation from 0 (over the sequences) of the error matrix is of the order $0.1 \%$ to $0.7 \%$ for the nine matrix elements mixing $Q, U$, and $V$, with a mean of $0.4 \%$, thus meeting the requirement of $1 \%$ (Section 1.1). For the $Q, U$, and $V$ to $I$ elements the values are less than $0.2 \%$, thus easily meeting the $10 \%$ requirement. As mentioned in Section 7.3.1, the determination of the instrument polarization is deferred to on-orbit and the model does not include it, resulting in the $I$ to $Q, U$, and $V$ errors being zero.

\subsubsection{Selection of Optimal Settings for LCP and RCP}

For a general polarization sequence that measures at least four non-degenerate states of polarization, the calibration ensures that the calibration errors are zero, assuming that the instrumental properties are known. 
However, as mentioned in Section 1.1, there is a requirement to be able to determine LCP and RCP from only two observations with less that 5\% crosstalk from $Q$ and $U$ across the FOV and with only the available motor positions. In this case it is not possible to calibrate out any linear-to-circular crosstalk and so the only way to meet the requirement is to rely on the uniformity of the optical properties across the FOV.

To determine if it is possible to satisfy these constraints, a search was performed. The result is that there are many such sequences, some of which keep not just the individual crosstalks but also the total linear-to-circular crosstalk below $3.8 \%$.

\subsection{Plans for On-Orbit Calibration}

As mentioned earlier, some parts of the calibration have been deferred to on-orbit. Also, the performance of the instrument will have to be checked and monitored for any aging-related effects or other types of changes.

\subsubsection{Items Deferred to On-Orbit}

There are two main items deferred to on-orbit tests: the telescope polarization, and the effective environment temperature to use to correct for the window depolarization. The latter is discussed in the following section.

The former should be straightforward to determine. Spatially and/or temporally averaged sunlight is almost perfectly unpolarized, especially in the continuum. It is thus possible to determine the telescope polarization by demanding that the incoming sunlight is unpolarized on average. The averaging may either be done using calmode or long-term averages in obsmode (possibly excluding active regions).

\subsubsection{Continuous Monitoring}

The instrument will change after launch. Experience with other missions indicates that some change and degradation is bound to happen. In the case of HMI, a likely change from a polarization point of view is an increase in the front-window absorptivity, as seen on the Michelson Doppler Imager (MDI) onboard the Solar and Heliospheric Observatory. The effect of this is that the window will absorb more energy, heat up, and cause the centerto-edge gradient to change. As discussed in Section 6.2.2, this has significant consequences and it is desirable to keep the gradient low by adjusting the setpoint of the window heaters. There are various ways that this degradation can be tracked.

The amount of energy supplied to the window heaters in order to maintain a specified temperature is monitored. From this a thermal model may be able to provide an estimate of the gradient as a function of window temperature.

Another effect of a temperature gradient is that the focus of the instrument will change, as was seen with MDI. In the absence of any other changes, the window temperature that keeps the focus fixed in time is thus likely to be the same one that will cause the gradient to remain constant.

Finally it may be possible to measure the depolarization by observing a sunspot and varying the window temperature. The polarization at best focus should then vary quadratically as seen in Figure 15, with the maximum indicating the desired operating temperature. Assuming that a suitable spot is available, an attempt to do this will be made during commissioning, otherwise another time will have to be found. It is undesirable to perform this test frequently as it takes considerable time to execute. 


\section{Conclusion}

The HMI instrument has been designed to measure full Stokes $I, Q, U$, and $V$ for the full disk, for several wavelengths with $0.5^{\prime \prime}$ pixels.

We have demonstrated that the polarimetric efficiency of the instrument is almost perfect and allows for these measurements to be done with the required accuracy. We have also demonstrated that we have been able to build a polarimetric model that will allow us to calibrate the data independent of the details of the observing mode chosen and the environment.

Acknowledgements This work was supported by NASA Contract NAS5-02139 to Stanford University. The authors are grateful to Dick Shine, Bruce Lites, and Jack Harvey for critical advice and Tony Darnell for help with the design of the test equipment. The authors would also like to thank numerous engineers and other staff members at Stanford University and Lockheed Martin Solar and Astrophysics Laboratory including John Miles, Brett Allard, Dave Kirkpatrick, Glenn Gradwohl, Thomas Nichols, Noah Katz, Sebastien Couvidat, Richard Wachter, Rock Bush, Phil Scherrer, Todd Hoeksema, and Yang Liu, for designing the instrument, running tests, making the data system run smoothly, and helping with analysis. NCAR is supported by the National Science Foundation.

Open Access This article is distributed under the terms of the Creative Commons Attribution Noncommercial License which permits any noncommercial use, distribution, and reproduction in any medium, provided the original author(s) and source are credited.

\section{Appendix A: Models of Various Types of Optical Elements}

The following subsections collect the information about how the various types of optical elements are modeled.

\section{A.1. Polarizer Models}

The matrix of a linear polarizer $P$ with an angle $\theta$ and a transmission $t$ is given by:

$$
P(\theta, t)=\frac{t}{2}\left(\begin{array}{cccc}
1 & \cos 2 \theta & \sin 2 \theta & 0 \\
\cos 2 \theta & \cos ^{2} 2 \theta & \cos 2 \theta \sin 2 \theta & 0 \\
\sin 2 \theta & \cos 2 \theta \sin 2 \theta & \sin ^{2} 2 \theta & 0 \\
0 & 0 & 0 & 0
\end{array}\right) .
$$

\section{A.2. Depolarizer Model}

For the results presented, only a three-term model has been used:

$$
D_{3}\left(D_{Q}, D_{U}, D_{V}\right)=\operatorname{diag}\left(1, D_{Q}, D_{U}, D_{V}\right) .
$$

\section{A.3. Retarder Models}

The matrix of a retarder $W$ with angle $\theta$ and retardance $r$ (in waves) is given by:

$$
W(\theta, r)=\left(\begin{array}{cccc}
1 & 0 & 0 & 0 \\
0 & c^{2}+s^{2} \cos \delta & c s(1-\cos \delta) & -s \sin \delta \\
0 & c s(1-\cos \delta) & s^{2}+c^{2} \cos \delta & c \sin \delta \\
0 & s \sin \delta & -c \sin \delta & \cos \delta
\end{array}\right),
$$

where $c=\cos 2 \theta, s=\sin 2 \theta$, and $\delta=2 \pi r$. 
A problem with Equation (10) is that $\theta$ becomes poorly defined as $r$ gets smaller, causing large spurious variations in $\theta$ in the presence of noise, as well as convergence problems. This can be avoided by using an alternate expression:

$$
W_{M}\left(M_{Q V}, M_{U V}\right)=W(\theta, r),
$$

where, formally, $M_{Q V}=-\sin 2 \theta \sin r$ and $M_{U V}=\cos 2 \theta \sin r$. In reality one avoids the calculation of $\theta$ and $r$ and calculates the other matrix elements and derivatives directly.

Another problem with Equation (10) is that it is only valid for normal incidence. For non-zero incidence angle the retardance has to be modified, with the result that

$$
W_{I}(\theta, r, \alpha, \beta)=W\left(\theta, r^{\prime}\right),
$$

where

$$
r^{\prime}=r\left(1+\alpha^{2} \cos (2(\beta-\theta)) / 2\right),
$$

$\alpha$ is the incidence angle and $\beta$ is the angle between the horizontal and the direction from the center of the field to the point of interest. In other words, $\beta-\theta$ is the angle between the fast axis of the waveplate and the direction from the center of the field to the point of interest. It is important that $r$ is the true retardance (e.g. 10.25 waves not 0.25 waves). Also note that the sign of the correction term is different for other waveplate materials.

\section{A.4. Combined Models}

If an optical element has a varying fast-axis direction along the ray path, the resulting Mueller matrix can not, in general, be described as a simple retarder, as given above. If there is also an amount of depolarization due to, for example, variation of the retardance direction or magnitude over the beam, the Mueller matrix is further complicated. This Mueller matrix

$$
M_{6}\left(M_{Q Q}, M_{Q U}, M_{Q V}, M_{U U}, M_{U V}, M_{V V}\right)=\left(\begin{array}{cccc}
1 & 0 & 0 & 0 \\
0 & M_{Q Q} & M_{Q U} & M_{Q V} \\
0 & M_{Q U} & M_{U U} & M_{U V} \\
0 & -M_{Q V} & -M_{U V} & M_{V V}
\end{array}\right),
$$

which captures an average of an arbitrary set of retarders, is useful in that case, although it may be noted that it has more degrees of freedom than needed, in that $M_{V V}=M_{Q Q}+$ $M_{U U}-1$ for an arbitrary combination of retarders.

\section{Appendix B: Polarization Calibration Sequences}

Two main sequences have been used for the calibration, the so-called short and long sequences. For each of a number of PCU configurations, one or more simple instrument sequences are taken. These are designed to measure simple polarization states with nominal waveplate properties. In other words they have been kept fixed over time and use initial guesses for the zero points etc. (rather than correcting them based on later information). Angles given below are in degrees and given relative to the numbers in Section 2.3. Also note that the PCU angles are nominal and refer to the LMSAL configuration where the bottom of the instrument and the bottom of the PCU are parallel. For the GSFC/ASO case $90^{\circ}$ has to be added to the PCU angles.

Standard sequences: 
- Single linear: Two darks and roughly $I+Q$ (waveplates at $0^{\circ}, 357^{\circ}$, and $45^{\circ}$ ).

- Double linear: Two darks and roughly $I \pm Q$ (above and $0^{\circ}, 357^{\circ}$, and $0^{\circ}$ ).

- Sequence A: Two darks, a four position sequence, a dark.

$\begin{array}{rrrrr}P L 1 & 0 & 0 & 23 & 23 \\ P L 2 & 357 & 357 & 357 & 357 \\ P L 3 & 36 & 9 & 54 & 81\end{array}$

- Sequence C: Two darks, a six-position sequence, a dark.

$\begin{array}{rrrrrrr}P L 1 & 0 & 0 & 23 & 23 & 0 & 0 \\ P L 2 & 357 & 357 & 357 & 357 & 357 & 357 \\ P L 3 & 45 & 0 & 45 & 0 & 23 & 68\end{array}$

- Sequence AC: Two darks, a four-position sequence, a six-position sequence, a dark.

$\begin{array}{rrrrrrrrrrr}P L 1 & 0 & 0 & 23 & 23 & 0 & 0 & 23 & 23 & 0 & 0 \\ P L 2 & 357 & 357 & 357 & 357 & 357 & 357 & 357 & 357 & 357 & 357 \\ P L 3 & 36 & 9 & 54 & 81 & 45 & 0 & 45 & 0 & 23 & 68\end{array}$

- Short combo: Two darks, the following sequence of waveplate angles, and a dark.

$\begin{array}{rrrrrrrrrr}P L 1 & 0 & 120 & 240 & 0 & 0 & 0 & 0 & 120 & 240 \\ P L 2 & 357 & 357 & 357 & 117 & 237 & 357 & 357 & 117 & 237 \\ P L 3 & 0 & 0 & 0 & 0 & 0 & 120 & 240 & 120 & 240\end{array}$

- Long combo: Two darks, the following sequences of waveplate angles, and a dark.

$\begin{array}{rrrrrrrrrrrr}P L 1 & 0 & 60 & 120 & 180 & 240 & 300 & 0 & 0 & 0 & 0 & 0 \\ P L 2 & 357 & 357 & 357 & 357 & 357 & 357 & 57 & 117 & 177 & 237 & 297 \\ P L 3 & 0 & 0 & 0 & 0 & 0 & 0 & 0 & 0 & 0 & 0 & 0 \\ P L 1 & 0 & 0 & 0 & 0 & 0 & 60 & 120 & 180 & 240 & 300 & \\ P L 2 & 357 & 357 & 357 & 357 & 357 & 57 & 117 & 177 & 237 & 297 & \\ P L 3 & 60 & 120 & 180 & 240 & 300 & 60 & 120 & 180 & 240 & 300 & \end{array}$

Note that sequences $\mathrm{A}$ and $\mathrm{C}$ are both complete polarization sequences able to determine $I, Q, U$, and $V$, similar to those intended for normal use.

In addition, sequences to monitor the stability of the setup are used:

- Short clear: Single linear with PCU out.

- Long clear: Double linear with PCU out.

B.1. Short Sequence (75 images and 65 darks)

- Short clear

- Without PCU: Sequence A

- Short clear

- Polarizer at $0^{\circ}$, retarder at $90^{\circ}, 150^{\circ}, 210^{\circ}, 270^{\circ}, 330^{\circ}, 30^{\circ}$ : Single linear

- Short clear

- Polarizer only at $0^{\circ}, 60^{\circ}, 120^{\circ}, 180^{\circ}, 240^{\circ}, 300^{\circ}$ : Single linear

- Short clear 
- Polarizer only at $0^{\circ}, 120^{\circ}, 240^{\circ}$ : Short combo and short clear

- Polarizer at $0^{\circ}$, retarder at $\mp 45^{\circ}$ : Short combo and short clear

- Without PCU: Sequence A

- Short clear

B.2. Long Sequence (192 images and 70 darks)

- Long clear

- Without PCU: Sequence AC

- Long clear

- Polarizer at $0^{\circ}$, retarder at $90^{\circ}, 150^{\circ}, 210^{\circ}, 270^{\circ}, 330^{\circ}, 30^{\circ}$ : Double linear

- Long clear

- Polarizer only at $0^{\circ}, 60^{\circ}, 120^{\circ}, 180^{\circ}, 240^{\circ}, 300^{\circ}$ : Double linear

- Long clear

- Polarizer only at $0^{\circ}, 120^{\circ}, 240^{\circ}$ : Long combo and long clear

- Polarizer at $0^{\circ}$, retarder at $\mp 45^{\circ}$ : Long combo and long clear

- Without PCU: Sequence AC

- Long clear

\section{References}

Beck, C., Schlichenmaier, R., Collados, M., Bellot Rubio, L., Kentischer, T.: 2005, A polarization model for the German vacuum tower telescope from in situ and laboratory measurements. Astron. Astrophys. 443, 1047 - 1053. doi:10.1051/0004-6361:20052935.

Beck, C., Schmidt, W., Kentischer, T., Elmore, D.: 2005, Polarimetric littrow spectrograph - instrument calibration and first measurements. Astron. Astrophys. 437, 1159-1167. doi:10.1051/0004-6361: 20052662.

Borrero, J.M., Tomczyk, S., Norton, A., Darnell, T., Schou, J., Scherrer, P., Bush, R., Liu, Y.: 2007, Magnetic field vector retrieval with the helioseismic and magnetic imager. Solar Phys. 240, 177-196. doi:10.1007/s11207-006-0219-x.

Collados, M.V.: 2003, Stokes polarimeters in the near-infrared. In: S. Fineschi (ed.) SPIE Conference Series, 4843, 55 - 65. doi:10.1117/12.459370.

Etzel, S.M., Rose, A.H., Wang, C.M.: 2000, Dispersion of the temperature dependence of the retardance in $\mathrm{SiO}_{2}$ and $\mathrm{MgF}_{2}$. Appl. Opt. 39, 5796-5800. doi:10.1364/AO.39.005796.

Ichimoto, K., Lites, B., Elmore, D., Suematsu, Y., Tsuneta, S., Katsukawa, Y., Shimizu, T., Shine, R., Tarbell, T., Title, A., Kiyohara, J., Shinoda, K., Card, G., Lecinski, A., Streander, K., Nakagiri, M., Miyashita, M., Noguchi, M., Hoffmann, C., Cruz, T.: 2008, Polarization calibration of the solar optical telescope onboard Hinode. Solar Phys. 249, 233 - 261. doi:10.1007/s11207-008-9169-9.

Kuhn, J.R., Balasubramaniam, K.S., Kopp, G., Penn, M.J., Dombard, A.J., Lin, H.: 1994, Removing instrumental polarization from infrared solar polarimetric observations. Solar Phys. 153, 143-155. doi:10.1007/BF00712497.

Norton, A.A., Graham, J.P., Ulrich, R.K., Schou, J., Tomczyk, S., Liu, Y., Lites, B.W., López Ariste, A., Bush, R.I., Socas-Navarro, H., Scherrer, P.H.: 2006, Spectral line selection for HMI: a comparison of Fe I $6173 \AA$ and Ni I 6768 A. Solar Phys. 239, 69-91. doi:10.1007/s11207-006-0279-y.

Schou, J., Scherrer, P.H., Bush, R.I., Wachter, R., Couvidat, S., Rabello-Soares, M.C., et al.: 2010, The helioseismic and magnetic imager instrument design and calibration. Solar Phys., in press.

Selbing, J.: 2005, SST polarization model and polarimeter calibration. Master's thesis, Stockholm University, Stockholm, Sweden.

Skumanich, A., Lites, B.W., Martinez Pillet, V., Seagraves, P.: 1997, The calibration of the advanced stokes polarimeter. Astrophys. J. Suppl. 110, 357. doi:10.1086/313004.

Wachter, R., Schou, J., Rabello-Soares, M.C., Miles, J.W., Duvall, T.L. Jr., Bush, R.I.: 2010, Image quality of the helioseismic and magnetic imager. Solar Phys., in press. 